\title{
A multi-material flow solver for high speed compressible flows
}

A. Kapahi, C.-T. Hsiao, and G. L. Chahine

DYNAFLOW, INC.

www.dynaflow-inc.com

\begin{abstract}
This paper describes a three-dimensional Eulerian-Lagrangian method for the modeling and simulation of high-speed multi-material dynamics. The equations for conservation of mass, momentum, and energy are solved on a fixed Cartesian grid using a fully conservative higher order MUSCL scheme. The dilatational response of each material is handled using a suitable equation of state. The embedded interfaces are handled using a mixed-cell approach. This approach uses an Eulerian treatment for the computational cells away from the interface and a Lagrangian treatment for the cells including interface elements, resulting in a fully conservative method for multi-material interactions. The method has shown capability to resolve and capture non-linear waves such as shock waves, rarefaction waves, and contact discontinuities in complex geometries. This work mainly emphasizes the handling of shockwave interaction with bubbles, bubbly media, and multi-fluid interfaces in a compressible flow framework. Several numerical examples are shown to demonstrate the validity and robustness of the method.
\end{abstract}

Keywords: Compressible Flows, Mixed Cell, Conservation, MUSCL scheme, Shock waves, interfaces

\section{Introduction}

The understanding of high speed flow phenomena is critical to many applications such as blast wave interactions with structures [2], underwater explosions [3], munitions-target interactions [4], detonation shock dynamics [5], dynamics of shaped charges[6], ... etc. 
These interactions involve the presence of non-linear waves such as shock waves, rarefaction waves, and detonation waves resulting in localized high intensity phenomena such as large deformation of the interface, fragmentation, and shattering.

The methods of choice for solving these problems can be mainly categorized into Eulerian and Lagrangian methods [4]. Both Lagrangian and Eulerian frameworks have been identified with certain advantages and limitations and take different paths in formulating large deformation problems $[4,7,8]$. Flow solvers based on a Lagrangian formulation, such as in EPIC [9] and LS-DYNA [10], have the material interface attached to the mesh, which is used to follow the deformation. Those based on an Eulerian formulation, such as HULL [11] and CTH [12], use a fixed mesh and the deformed material flows through the mesh. The Lagrangian methods have to contend with mesh entanglement and the burden of mesh management encountered frequently in large deformation problems making them cumbersome to handle $[4,8]$. On the other hand, Eulerian methods, which use level sets and ghost fluid approaches, have issues of mass conservation resulting in loss/gain of mass of material while undergoing large deformation $[6,13,14,15,16]$. There are some other methods such as Smooth Particle Hydrodynamics [17], which is a variant of Lagrangian method, designed to handle severe deformations and where the location of the material interface is not as important and Arbitrary Lagrangian Eulerian methods, which optimize the solution by allowing the mesh inside the domain to move arbitrary while mesh attached to the interface moves precisely to track the interface $[18,19,20,21]$.

In the present work, a high-order compressible fluid solver, 3DYNAFS-COMP ${ }^{\circledR}$, based on a mixed cell (Eulerian-Lagrangian) approach [19,22,23] is developed to handle shock wave interactions with fluid or material interfaces. This approach uses a Lagrangian treatment for cells containing a material interface and an Eulerian treatment for cells away from material interfaces. A re-map procedure is employed to map the Lagrangian solution back to the 
Eulerian grid. Note that though this approach is called mixed cell method, it is different from mixture theory approaches where averaged properties are used for the computational cells containing more than one material [5]. The present mixed cell approach still treats each material separately but takes the benefit from both the Eulerian and Lagrangian treatments and can handle large deformation problems without having issues with the conservation of physical quantities.

As discussed above, the treatment of the interfaces is the key component in handling a multimaterial compressible flow solver. This paper emphasis is on bubble dynamics during which the air/water interface undergoes severe deformation. Moreover, shock wave interactions with the air/water interface could result in shattering of the bubble(s). The high order method developed in this work addresses the following key matters involved with interface treatment[24]:

1. The interfaces are tracked using an Eulerian-Lagrangian approach and only the computational cells containing material interfaces are treated in a fully Lagrangian manner. This procedure obviates extensive re-meshing and can handle large deformations of the interface. A re-map procedure, which involves mesh management, is applied only on a lower order dimension (i.e. on a curve for a $2 \mathrm{D}$ object, or a surface for a 3D object) and is less computationally expensive than in a fully Lagrangian approach where the distortion of a material interface can require smaller and smaller time-steps leading to termination of the simulation. In other words, the Lagrange and re-map procedure will be required only for the cells defining the interface and not for the whole domain.

2. The issue of conservation is a key challenge in high-speed multi-material flows. Lagrangian methods, though fully conservative, have to implement erosion techniques 
to delete material points as the deformation increases. On the other hand, Eulerian methods facilitate handling of large deformations at the cost of being nonconservative at the material interface. The mixed approach used in this work combines both Eulerian and Lagrangian techniques by carefully treating the material interface resulting in a fully conservative procedure capable of handling large deformations.

3. A mixture based approach where the flow quantities [5] are averaged in the computational cells containing more than one material can be catastrophic for materials with large differences in impedance. Moreover, an averaging approach also requires some sort of averaged equation of state to compute the dilatational response. These procedures may give a physical solution for materials with similar properties but could lead to erroneous solutions for problems having more than two materials or a pair of stiff materials. The developed Eulerian-Lagrangian approach has been shown to work well for air and water interfaces (stiff pair) for a variety of problems and matches well with existing established flow solvers [23].

With these issues well addressed, the present method establishes a framework to handle shock interaction with material interfaces. It can also be used to prescribe a homogeneous mixture field in a given region, treat it as a block of mixed cells and then investigate its evolution and multi-species dynamics. This is an advantage over other methods, which can treat embedded object only as single-phase materials and makes the current algorithm more flexible. Another main advantage of the present software is that it can also address complex two-phase flows through seamless coupling with other companion modules such as a Discrete Singularity Model (DSM) [25]to handle complex bubble cloud problems. This will be a 
subject of separate publications. A slight disadvantage of the current method is that the interface is not sharp and its precision depends on the selected grid cell sizes.

The details of the governing equations, numerical methods, interface treatment, and application on a few numerical examples showing robustness of the method are presented in the following sections.

\section{Governing Equations}

The governing equations in the framework presented above, describe a set of hyperbolic conservation laws [26]. Cast in Cartesian coordinates, the governing equations for the conservation of mass, momentum, and energy take the following form:

$$
\begin{gathered}
\frac{\partial Q}{\partial t}+\frac{\partial F}{\partial x}+\frac{\partial G}{\partial y}+\frac{\partial H}{\partial z}=0, \\
Q=\left[\begin{array}{c}
\rho \\
\rho u \\
\rho v \\
\rho w \\
\rho E
\end{array}\right], F=\left[\begin{array}{c}
\rho u \\
\rho u^{2}+p \\
\rho u v \\
\rho u w \\
u(\rho E+p)
\end{array}\right], G=\left[\begin{array}{c}
\rho v \\
\rho u v \\
\rho v^{2}+p \\
\rho v w \\
v(\rho E+p)
\end{array}\right], H=\left[\begin{array}{c}
\rho w \\
\rho u w \\
\rho v w \\
\rho w^{2}+p \\
w(\rho E+p)
\end{array}\right] .
\end{gathered}
$$

In Equation (1), $Q$ contains the primitive variables and $F, G$, and $H$ contain the fluxes in the $x, y$, and $z$ directions respectively. The variables $u, v$, and $w$ are the velocities in the $x, y$, and $z$ directions respectively, $\rho$ is the material density, $E$ is the specific total energy of the material, and $p$ is the pressure. The system is closed by using a suitable equation of state, which defines the pressure as a function of the specific internal energy and the density. In this paper we consider air/water interfaces. An ideal gas equation is used for air [28]

$$
p=(\gamma-1) \rho e,
$$

where $\gamma=1.4$. The specific internal energy $e$ can be defined as 


$$
e=E-\frac{1}{2}\left(u^{2}+v^{2}+w^{2}\right)
$$

One of two potential equations of state - Tait law and Tillotson [29] - are used for water. The expression for the Tait law is

$$
p=p_{0}+B\left[\left(\rho / \rho_{0}\right)^{n}-1\right]
$$

where the constants $n=7.15, \quad B=3 \times 10^{8} \mathrm{~Pa}$, and $p_{0}=10^{5} \mathrm{~Pa}$ and $\rho_{0}=1000 \mathrm{Kg} / \mathrm{m}^{3}$ are reference pressure and density respectively. Note that Equation (4) does not explicitly invoke the energy. Therefore, materials described using the Tait law do not require the solution of the energy equation. Tillotson gives a more complete equation of state for water, which can be written as

$$
p=p_{0}+\omega \rho\left(e-e_{0}\right)+A \mu+B \mu^{2}+C \mu^{3} ; \mu=\frac{\rho}{\rho_{0}}-1
$$

where the constants $\omega=0.28, A=2.2 \times 10^{10}, B=9.54 \times 10^{10}, C=1.457 \times 10^{11}$, and $p_{0}=$ $10^{5} \mathrm{~Pa}, e_{0}=3.542 \times 10^{5} \mathrm{~J} / \mathrm{Kg}$ and $\rho_{0}=1000 \mathrm{Kg} / \mathrm{m}^{3}$ are the reference pressure, specific internal energy, and density respectively. The specific internal energy $e$, defined in Equation (3), can be used to deduce the temperature, $T$, of a given material using the following thermodynamic relation:

$$
d e=c_{v} d T+\left[\left.T \frac{\partial P}{\partial T}\right|_{\rho}-P\right] d\left(\frac{1}{\rho}\right),
$$

where is the specific heat at constant volume and $d$ is the differential operator. The term in the square bracket on the right hand side in equation (6) vanishes for ideal gas law where $P=\rho R T$, but will be important for non-ideal gases described for example by the Tillotson equation of state. For an ideal gas, the equation (6) can be written as: 


$$
d e=c_{v} d T
$$

\section{Numerical Methodology}

A stringent requirement for a compressible flow solver is to accurately capture non-linear waves such as shock waves, rarefaction waves, and contact discontinuities [24]. A typical finite difference scheme will generate considerable spurious oscillations at the shock interface [26]. These oscillations occur rapidly (within a few time steps) and often destabilize the numerical algorithm. Several schemes have been proposed to address the propagation of shock waves [30]. Early work based on artificial viscosity included extra terms in the finite difference-based discretized equation [31]. Approaches such as adding a viscous-like flux term, where the viscous coefficient is a complex function of the second derivative of the local pressure, have the objective of artificially increasing the viscous dissipation in regions of high pressure gradient. Although these methods are well suited for simple problems where the various parameters can be adjusted to provide the proper amount of dissipation to stabilize the solution without excessively smearing the overall computed quantities, they are difficult to optimize for general problems. Other shock capturing approaches are based on the local decomposition of the equations into characteristic flux vectors[26,31].

Initial value problems which involve discontinuities in the solution are known as Riemann problems [26] which can be best solved by Godunov-type methods [32]. These differ from conventional finite difference methods constructed from Taylor series approximations. Their main advantage is that they are robust and accurately capture the location of discontinuities such as shocks and contact surfaces. A good discussion of these methods is given in [26].

The present work uses a higher order predictor-corrector method based on the Monotonic Upwind Scheme for Conservation Law (MUSCL) [22] for pure cell calculations. In the predictor step, cell edge properties (density, velocity, energy) at the 'half step' are computed 
using the method of characteristics [22]. These properties are used to construct a Riemann problem at each cell edge. The procedure to handle the Riemann problem generates "regions" with different solutions. The computational cell edge properties are selected in accordance with the "region" in which the edge is located and these properties are used to compute the fluxes across the cell. Since this is a direction-by-direction procedure, these fluxes are shown for only the x direction. The fluxes of mass, $F^{\rho}$, momentum, $F^{v}$, and energy, $F^{E}$, at the cell edge face can be written for cell $i$, with $i+1 / 2$ corresponding to cell face location $(i+1$ is the neighboring cell) as:

$$
\begin{gathered}
F_{i+1 / 2}^{\rho}=A_{i+1 / 2} \rho_{i+1 / 2}^{g} u_{i+1 / 2}^{g}, \\
F_{i+1 / 2}^{u}=A_{i+1 / 2}\left[\rho_{i+1 / 2}^{g}\left(u_{i+1 / 2}^{g}\right)^{2}+p_{i+1 / 2}^{g}\right], \\
F_{i+1 / 2}^{E}=A_{i+1 / 2}\left[\rho_{i+1 / 2}^{g} u_{i+1 / 2}^{g} E_{i+1 / 2}^{g}+p_{i+1 / 2}^{g} u_{i+1 / 2}^{g}\right] .
\end{gathered}
$$

In Equations (8), the edge quantities, denoted by superscript $g$, are used to compute the fluxes. The variable $A_{i+1 / 2}$ represents the area at the cell edge defined as the product of $d y(i)$ and $d z(i)$, where $d y(i)$ and $d z(i)$ are the cell dimensions in $Y$ and $Z$ directions respectively.

The overall predictor-corrector method consists of a step to calculate the slopes of the primitive variables, a predictor step to apply the method of characteristic, and a corrector step involving the Riemann solver. This is a well-established algorithm and details corresponding to each step are provided in [22].

Once the density, energy, and pressure are updated at a given time step, the temperature can be computed by using first-order time-differencing expressions of Equations (6) and (7):

$$
T^{n+1}=\frac{e^{n+1}-e^{n}+c_{v} T^{n}+p^{n+1}\left[\frac{1}{\rho^{n+1}}-\frac{1}{\rho^{n}}\right]}{c_{v}+\omega \rho^{n+1} c_{v}\left[\frac{1}{\rho^{n+1}}-\frac{1}{\rho^{n}}\right]},
$$




$$
T^{n+1}=\frac{e^{n+1}-e^{n}+c_{v} T^{n}}{c_{v}} .
$$

In the equations (9) and (10), the superscript $n+1$ indicates the solution at the new time step and $n$ indicates the solution at the previous time step.

\subsection{Mixed Cell Treatment}

The standard MUSCL scheme is augmented by the mixed cell approach to handle material interfaces. In a scenario of multiple materials, the single fluid algorithm [22] initiates the solution by using average properties. The cells containing the material interfaces and their immediate neighbors known as "mixed cells" are revisited and re-solved using a fully conservative Lagrange-plus-remap step. In the Lagrange step, mixed cell edges are moved at local fluid velocities approximated by the previously computed Riemann slip velocity (corrector step) [22], while edges with pure cells on both sides are held fixed. Thus, mixed cells are treated from the Lagrange point of view, while adjacent pure cells have one edge that moves at the local fluid velocity and the second edge is stationary. During the re-map step, the convected flow is mapped back to the original grid. The process is shown schematically for a 1D problem in Figure 1, where the mixed cell contains an interface.

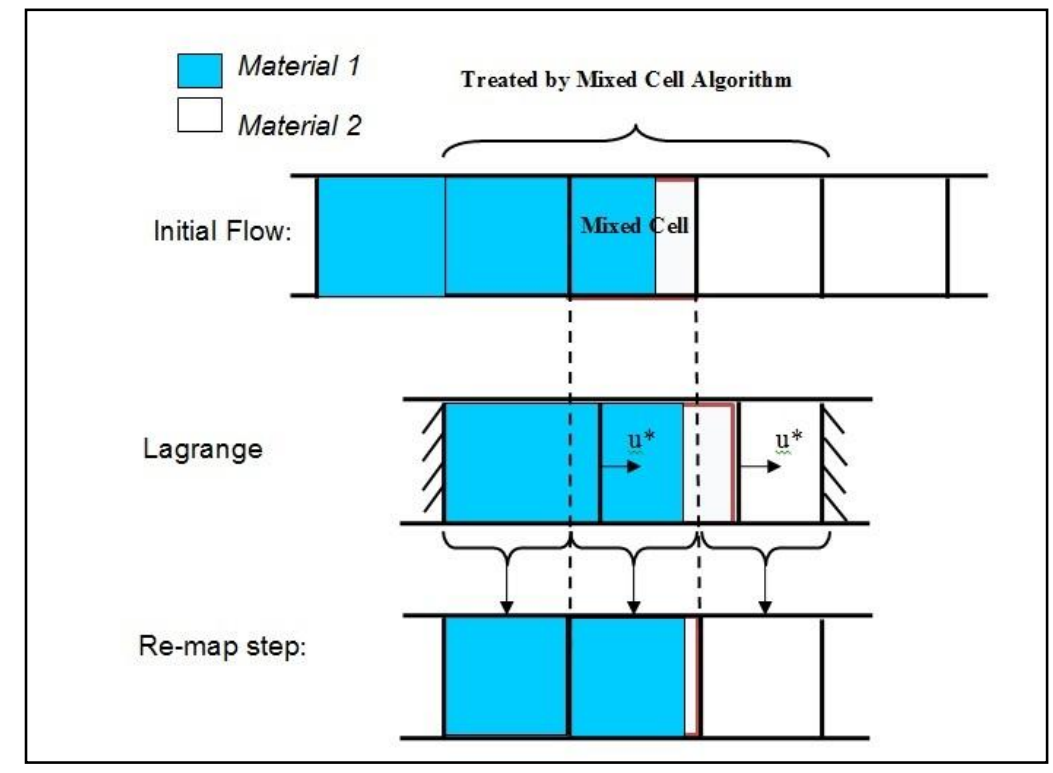


Figure 1. Sketch of the procedure used in the treatment of a mixed cell, which contains an interface. Mixed cells are advanced using a Lagrange step plus a re-map step.

If this cell was handled in a purely Lagrangian manner, the cell edges would move with an edge velocity, $u^{*}[22]$, as illustrated by the "Lagrange step". To maintain the original Eulerian grid, a "re-map step" is applied. This is a re-grid procedure, which brings back the fictitiously moved grid to the original location as illustrated by the dashed lines.

For a multi-dimensional configuration, the above procedure is repeated direction by direction. As the interface is not sharp and is defined within a single grid cell, the interface will be aligned with the mesh depending on the direction of application of the procedure. This is illustrated in Figure 2 by considering a circular interface in a 2D Cartesian mesh. For the computation in the $\mathrm{X}$-direction, the interface segments in each grid cell will be parallel to OY while for the Y-direction computations, the interface will be parallel to OX. Figure 2 (b) and Figure 2 (c) each highlight one of the mixed cells, which can be treated similar to a mixed cell in one dimension.

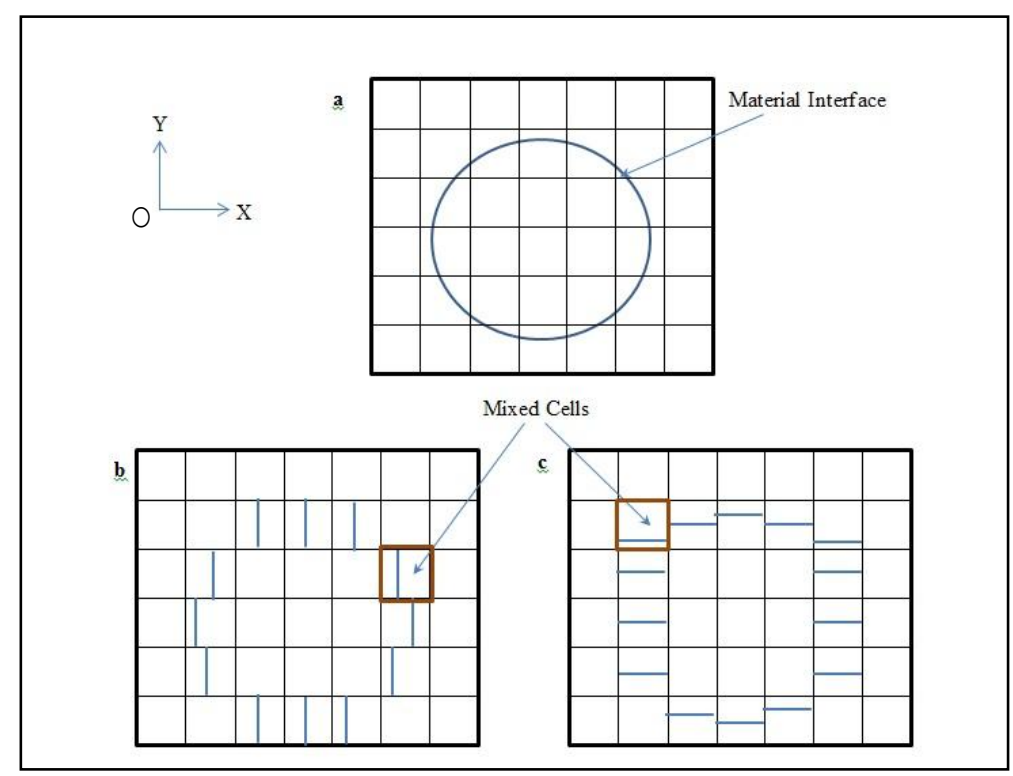


Figure 2. (a) Circular interface in a Cartesian mesh. (b) Interface as seen from the X-direction computations.

(c) Interface as seen from Y-direction computations. Each cell containing a line segment is a mixed cell.

\subsubsection{Lagrange Step}

\subsubsection{Governing Equations}

During the Lagrangian step, the volume, $V$, of a mixed cell can change. Cell volume expansion and conservation of mass, momentum, and energy during the Lagrange step can be written, with $\mathbf{U}$ being the velocity vector:

$$
\frac{D}{D t} \iiint d V=\nabla \cdot \mathbf{U} \iiint d V
$$

$$
\begin{aligned}
& \frac{D}{D t} \iiint \rho d V=0, \\
& \frac{D}{D t} \iiint \rho \mathbf{U} d V=-\nabla \cdot \iiint p \mathbf{I} d V, \\
& \frac{D}{D t} \iiint \rho E d V=-\nabla \cdot \iiint p \mathbf{U} d V .
\end{aligned}
$$

Equations (12) are nothing but the Euler hyperbolic equations (1) written in the material frame of reference. The operator $D / D t$ represents total derivative, i.e. derivative while moving with the material with the velocity $\boldsymbol{U} . \nabla \bullet$ represents the divergence operator. To track individual material property changes it is necessary to break down in these equations the quantities into their material-specific components. This necessitates dividing the change in volume and the pressure work among the individual materials. The total volume change can be simply stated as the sum of all individual material volumes as follows:

$$
d V=\sum_{m=1}^{M} d V^{m}
$$


where superscript $m$ represents the different materials. The individual elements volume changes can be related to the individual material masses, $M^{m}$, by:

$$
V^{m}=v^{m} M^{m},
$$

where $v^{m}$ is the specific volume of material $m$. Since $M^{m}$ is constant during a Lagrange step, the change in material $m$ volume, $d V^{m}$, can be written as:

$$
d V^{m}=M^{m} d v^{m}
$$

Introducing the material component sound speed, $c^{m}$, defined as:

$$
c^{m^{2}}=-v^{m^{2}} \frac{d p}{d v^{m}}
$$

and assuming that the change in pressure in the computational cell is the same for all individual materials, $d p=d p^{m}$, the equation (15) can be re-written as:

$$
d V^{m}=-\frac{d p^{m} V^{m}}{\rho^{m} c^{m^{2}}}
$$

Finally, using (17), the distribution of the volume change, can be written in terms of the material compressibility parameter [33], $\eta^{m}$ :

$$
\eta^{m}=\frac{d V^{m}}{d V}=\left(V^{m} \frac{d p^{m}}{\rho^{m} c^{m 2}}\right) /\left(V \frac{d p}{\rho c^{2}}\right),
$$

where $c$ is the sound speed in the mixed cell and $\alpha^{m}$ is the volume fraction corresponding to material $m$,

$$
c^{2}=\frac{\eta^{m} \rho^{m}}{\rho \alpha^{m}} c^{m^{2}}, \quad \alpha^{m}=\frac{V^{m}}{V} .
$$

The same procedure is applied to the momentum and energy conservation equations in (12) to obtain: 


$$
\begin{gathered}
\frac{D}{D t} \iiint \rho^{m} \mathbf{U} d V^{m}=-\mu^{m} \nabla \cdot \iiint p \mathbf{I} d V, \\
\frac{D}{D t} \iiint \rho^{m} E^{m} d V^{m}=-\eta^{m} p \nabla \cdot \iiint \mathbf{U} d V-\mu^{m} \mathbf{U} \nabla \cdot \iiint p \mathbf{I} d V,
\end{gathered}
$$

with

$$
\mu^{m}=\alpha^{m} \rho^{m} / \rho,
$$

where $\mu^{m}$ is the component $m$ mass fraction,

\subsubsection{Discretized Formulation}

The mixed cell volume, $V$, after the Lagrange step can be written in discretized format as follows:

$$
\hat{V}_{i}=V_{i}+\Delta V_{i} ; \quad \Delta V_{i}=\left(A_{i+\frac{1}{2}} u_{i+\frac{1}{2}}^{e}-A_{i-\frac{1}{2}} u_{i-\frac{1}{2}}^{e}\right) \Delta t
$$

Here and in what follows the ${ }^{\wedge}$ superscript denotes values at the end of this step. The subscript $i$ is the cell index and $i \pm 1 / 2$ represents the cell edge. The symbol $\Delta$ represents change in the subject quantity during the time interval $\Delta t$.In the equation (22), $u_{i+\frac{1}{2}}^{e}$ and $u_{i-\frac{1}{2}}^{e}$ are equal to $u^{*}$ for the moving edges and are equal to zero for the stationary edges.

The individual material volumes are given by:

$$
\hat{V}_{i}^{m}=V_{i}^{m}+\Delta V_{i} \eta_{i}^{m}
$$

Similarly, material specific continuity, momentum, and energy equation can be written as:

$$
\begin{gathered}
\hat{\rho}_{i}^{m} \hat{V}_{i}^{m}=\rho_{i}^{m} V_{i}^{m}, \\
\hat{\rho}_{i}^{m} \hat{V}_{i}^{m} \hat{u}_{i}=V^{m} \rho^{m}{ }_{i}^{m} u_{i}+\mu_{i}^{m}\left[\left(A_{i-\frac{1}{2}} p_{i-\frac{1}{2}}-A_{i+\frac{1}{2}} p_{i+\frac{1}{2}}\right)\right] \Delta \mathrm{t},
\end{gathered}
$$




$$
\begin{aligned}
\hat{\rho}_{i}^{m} \hat{V}_{i}^{m} \hat{E}_{i}^{m}= & \rho_{i}^{m} V_{i}^{m} E_{i}^{m}+\frac{\left(p_{i-\frac{1}{2}}+p_{i+\frac{1}{2}}\right)\left(u_{i-\frac{1}{2}} A_{i-\frac{1}{2}}-u_{i+\frac{1}{2}} A_{i+\frac{1}{2}}\right)}{2} \eta_{i}^{m}+ \\
& \frac{\left(u_{i-\frac{1}{2}} A_{i-\frac{1}{2}}+u_{i+\frac{1}{2}} A_{i+\frac{1}{2}}\right)\left(p_{i-\frac{1}{2}}-p_{i+\frac{1}{2}}\right)}{2} \mu_{i}^{m} .
\end{aligned}
$$

In the above expressions , $u_{i+\frac{1}{2}}$ and $u_{i-\frac{1}{2}}$ are equal to $u^{*}$ for the moving edges and are $u_{i+\frac{1}{2}}^{g}$ and $u_{i-\frac{1}{2}}^{g}$ respectively for the stationary edges. The updated material density is obtained from the mass conservation equation (24) as:

$$
\hat{\rho}_{i}^{m}=\frac{\rho_{i}^{m} V_{i}^{m}}{\hat{V}_{i}^{m}}
$$

The total density can then be computed using (27) as $\hat{\rho}_{i}=\sum_{m=1}^{M} \hat{\alpha}_{i}^{m} \hat{\rho}_{i}^{m}$. This can be used along with equation (25) to obtain the updated value of the velocity, which is assumed to be the same for all material components:

$$
\hat{u}_{i}=\frac{V_{i} \rho_{i} u_{i}+\left(A_{i-\frac{1}{2}} p_{i-\frac{1}{2}}-p_{i+\frac{1}{2}} A_{i+\frac{1}{2}}\right) \Delta t}{\hat{V}_{i} \hat{\rho}_{i}} .
$$

Finally the new value of the material energy using the above and Equation (26):

$$
\begin{gathered}
\hat{E}_{i}^{m}=\frac{E_{i}^{m} \rho_{i}^{m} V_{i}^{m}}{\hat{\rho}_{i}^{m} \hat{V}_{i}^{m}}+\frac{\left(p_{i-\frac{1}{2}}+p_{i+\frac{1}{2}}\right)\left(u_{i-\frac{1}{2}} A_{i-\frac{1}{2}}-u_{i+\frac{1}{2}} A_{i+\frac{1}{2}}\right) \eta_{i}^{m}}{2 \hat{\rho}_{i}^{m} \hat{V}_{i}^{m}} \Delta t \\
+\frac{\left(u_{i-\frac{1}{2}} A_{i-\frac{1}{2}}+u_{i+\frac{1}{2}} A_{i+\frac{1}{2}}\right)\left(p_{i-\frac{1}{2}}-p_{i+\frac{1}{2}}\right) \mu_{i}^{m}}{2 \hat{\rho}_{i}^{m} \hat{V}_{i}^{m}} \Delta t .
\end{gathered}
$$




\subsubsection{Re-map Step}

The re-map step is a re-gridding procedure and projects the Lagrange step results back onto the original mesh as is shown in Figure 1. This entails:

1. determining the volume to be moved between adjacent cells to restore the original grid,

2. splitting this volume into material components, and

3. computing the average mass, momentum, and energy in the reconstituted cells.

The volume to be moved across each edge is computed as:

$$
v_{i+1 / 2}=A_{i+1 / 2} u_{i+1 / 2}^{f} \Delta t
$$

The Simple Line Interface Computation (SLIC) algorithm [34] is used to decompose $v$ into material-specific volume components, $v^{m}$, depending on neighboring materials. Each material volume, $v^{m}$, is then used to determine the volume flux of that material, and is given by

$$
v_{i+1 / 2}^{m}=v^{m} \operatorname{sign}\left(u_{i+1 / 2}^{f}\right)
$$

The computed volume fluxes are used to map the Lagrangian solution back to the original

grid. The fluxes for the re-map step: $F^{\rho^{m}}$ for the mass, $F^{u}$ for the momentum, and $F^{E^{m}}$ for the energy, can be written as:

$$
\begin{gathered}
\left(F_{i+1 / 2}^{\rho^{m}}\right)^{\text {remap }}=v_{i+1 / 2}^{m} \rho_{I}^{m}, \\
\left(F_{i+1 / 2}^{u}\right)^{\text {remap }}=v_{i+1 / 2}^{m} \rho_{I} u_{I}, \\
\left(F_{i+1 / 2}^{E^{m}}\right)^{\text {remap }}=v_{i+1 / 2}^{m} \rho_{I}^{m} E_{I}^{m} .
\end{gathered}
$$

The value of the subscripts, $I$, in equations (32) is decided by following relation: 


$$
I=\left\{\begin{array}{lll}
i+1, & \text { for } & u_{i+1 / 2}^{f} \leq 0 \\
i, & \text { for } & u_{i+1 / 2}^{f}>0
\end{array}\right\} .
$$

The final cell properties (volume fraction, density and energy) given by superscript ${ }^{n+1}$ after the re-map procedure can be determined from:

$$
\begin{gathered}
\alpha_{i}^{m^{n+1}}=\frac{\hat{\alpha}_{i}^{m} \hat{V}_{i}+\left(v_{i-\frac{1}{2}}^{m}-v_{i+\frac{1}{2}}^{m}\right)}{V_{i}^{n+1}}, \\
\rho_{i}^{m^{n+1}}=\frac{\hat{\alpha}_{i}^{m} \hat{V}_{i} \hat{\rho}_{i}^{m}+\left(\left(F_{i-\frac{1}{2}}^{\rho^{m}}\right)^{\text {remap }}-\left(F_{i+\frac{1}{2}}^{\rho^{m}}\right)^{\text {remap }}\right)}{\alpha_{i}^{m^{n+1}} V_{i}^{n+1}}, \\
E_{i}^{m^{n+1}}=\frac{\hat{\alpha}_{i}^{m} \hat{V}_{i} \hat{\rho}_{i}^{m} \hat{E}_{i}^{m}+\left(\left(F_{i-\frac{1}{2}}^{E^{m}}\right)^{\text {remap }}-\left(F_{i+\frac{1}{2}}^{E^{m}}\right)^{\text {remap }}\right)}{\alpha_{i}^{m^{n+1}} V_{i}^{n+1} \rho_{i}^{m^{n+1}}} .
\end{gathered}
$$

After the properties of each material on the original grid are obtained at the re-map step, the average properties in a cell containing $M$ materials can be calculated.

The equivalent density is volume averaged and can be written as:

$$
\rho=\sum_{m=1}^{M} \alpha^{m} \rho^{m}
$$

The energy is mass averaged and can be written as:

$$
E=\frac{1}{\rho} \sum_{m=1}^{M} \alpha^{m} \rho^{m} E^{m}
$$

The average value of the density is used to compute the final velocity:

$$
u_{i}^{n+1}=\frac{\hat{V}_{i}^{n} \hat{\rho}_{i}^{n} \hat{u}_{i}^{n}+\left(F_{i-\frac{1}{2}}^{u}\right)^{\text {remap }}-\left(F_{i+\frac{1}{2}}^{u}\right)^{\text {remap }}}{V_{i}^{n+1} \rho_{i}^{n+1}} .
$$

The sound speed as obtained as in (19) is averaged from all components with the final expression written as: 


$$
c^{2}=\frac{1}{\rho \sum_{m=1}^{M} \frac{\alpha^{m}}{\rho^{m} c^{m 2}}} .
$$

The material specific internal energy and density are used to find the material specific pressure. A pressure equilibrium algorithm $[23,33]$ is used to equilibrate the pressure so that pressure continuity can be achieved at the material interface. This procedure adjusts the material volume fraction in a mixed cell, while holding the energy constant, with a constrain on volume fraction $\sum_{m=1}^{M} f^{m}=1$, until all materials are at the same pressure. In the case of no solution, the single value of pressure in a mixed cell is computed based on the change in volume and the final expression can be obtained by substituting $d p^{m}=p-p^{m}$ in equation (17):

$$
p=\frac{\sum_{m=1}^{M} \frac{p^{m} \alpha^{m}}{\rho^{m} c^{m 2}}}{\sum_{m=1}^{M} \frac{\alpha^{m}}{\rho^{m} c^{m 2}}} .
$$

The material specific quantities $\rho^{m}, e^{m}$, and the total pressure $p$ from the above equations are used to compute the temperature of each individual material, $T^{m}$ based on Equations (9) and (10) and the mass-averaged temperature in a mixed cell can be written as:

$$
T=\frac{1}{\rho} \sum_{m=1}^{M} \alpha^{m} \rho^{m} T^{m}
$$

These average properties are then used to advance the solution as in a single fluid problem.

\section{Benchmarking and Verification}

This section will show some typical numerical problems simulated using 3DYNAFS-COMP ${ }^{\odot}$. The first cases consider classical examples to validate the implementation and determine 
accuracy. The later examples address more complex problems related to bubble dynamics and two-phase bubbly flow. Gravity and surface tension effects are ignored in these examples. Introduction of gravity is trivial, while surface tension requires additional considerations.

Unless otherwise stated, all example problems shown below are solved using a CFL number of 0.45. The CFL number is calculated here as follows:

$$
\mathrm{CFL}=\frac{\left(u_{\max }+c\right) \Delta t}{\Delta x}
$$

where $u_{\max }$ is the maximum value of all absolute local material speeds, $c$ is the sound speed, $\Delta t$ and $\Delta x$ are the time step and the grid spacing respectively. The selected value of the CFL is used to compute the adaptive time step duration the computation.

All problems presented below were solved on a single processor. Parallelization using OpenMP and MPI is the subject of ongoing work.

\subsection{Shock tube problem}

The shock tube problem is a standard test case to benchmark a compressible flow solver [26]. A shock tube is comprised of two sections separated by a diaphragm with a high-pressure gas in one section and a low-pressure gas in the other. The sudden removal of the diaphragm results in three characteristics waves: a shock wave, a rarefaction wave, and a contact discontinuity. The high-pressure shock wave propagates into the initially low-pressure region resulting in a sharp front whereas a weaker rarefaction wave moves into the initially highpressure region. This dynamics can be simulated using the numerical framework described above. Two example cases are presented here, one corresponding to a weak shock (Sod's shock tube problem) and the second to a strong shock [26]. The initial conditions 
corresponding to each case are given in Table 1. The ideal gas law given in Equation (2) is used to compute the pressures in both cases.

\begin{tabular}{|c|c|c|c|c|c|c|}
\hline \multirow{2}{*}{ Case } & \multicolumn{2}{|c|}{\begin{tabular}{c} 
Normalized \\
\multicolumn{2}{|c|}{ Pressure }
\end{tabular}} & \multicolumn{2}{c|}{ Dormalized } & \multicolumn{2}{c|}{ Normalized } \\
\cline { 2 - 7 } & Left & Right & Left & Right & Left & Right \\
\hline Weak & 1.0 & 0.1 & 1.0 & 0.125 & 0.0 & 0.0 \\
Shock & & & & & & \\
Strong & 100.0 & 1.0 & 10.0 & 1.0 & 0.0 & 0.0 \\
Shock & & & & & & \\
\hline
\end{tabular}

Table 1. Initial conditions for the two shock tube problems discussed below: a weak and a strong shock.

The spatial distributions of the pressure, density and temperature at an instant before the waves reach the boundaries are compared with the exact solution in Figure 3 and Figure 4 respectively. Both problems are simulated using 200 grid points in the X-direction unit length. The figures clearly show that all three waves are properly and accurately captured and the results are in excellent agreement with the exact solution 26 for both the weak and strong shock cases. At the instants shown in Figure $3(t=0.25)$ and Figure $4(t=0.08)$, the shock wave (S) is located at $\mathrm{X}=0.93$ for the weak case and at $\mathrm{X}=0.89$ for the strong case. The contact discontinuity (C) moves into the originally low-pressure region of the shock tube. The pressure is continuous across this contact discontinuity $(\mathrm{C})$ located at $\mathrm{X}=0.73$ in Figure 3 and at $\mathrm{X}=0.81$ in Figure 4, while the density and temperature are discontinuous, since by definition, contact discontinuities are surfaces separating two regions with different densities across which there is no cross flow and the pressure is in equilibrium. The rarefaction wave 
(R), which is not a sharp discontinuity and represents a gradual change in the flow quantities has its head located at about $\mathrm{X}=0.2$ for both the weak and strong shock cases as shown in Figure 3 and Figure 4 respectively. At the observation time corresponding to Figure 3 and Figure 4 respectively, the tail of the rarefaction wave $(R)$ is located at $X=0.48$ for the weak shock and at $\mathrm{X}=0.56$ for the strong shock.

The grid used for this problem was selected by conducting a grid/CFL independent study. The results from this study are summarized in Figure 5. The pressure variations in the domain are shown at $t=0.25$ for different space resolution $(\mathrm{CFL}=0.45$, Figure $5 \mathrm{a})$ and time resolution (CFL $<1$, Figure $5 b)$. The results clearly show that 200 grid points and a CFL of 0.45 are good selections for a converged solution. For this reference condition, the serial computations took 90 CPU seconds on a $3.33 \mathrm{GHz}$ quad core computer. 
a

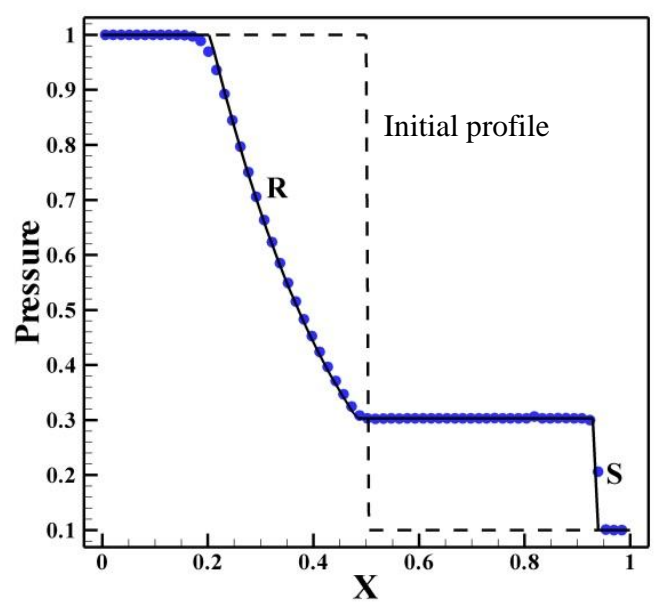

b

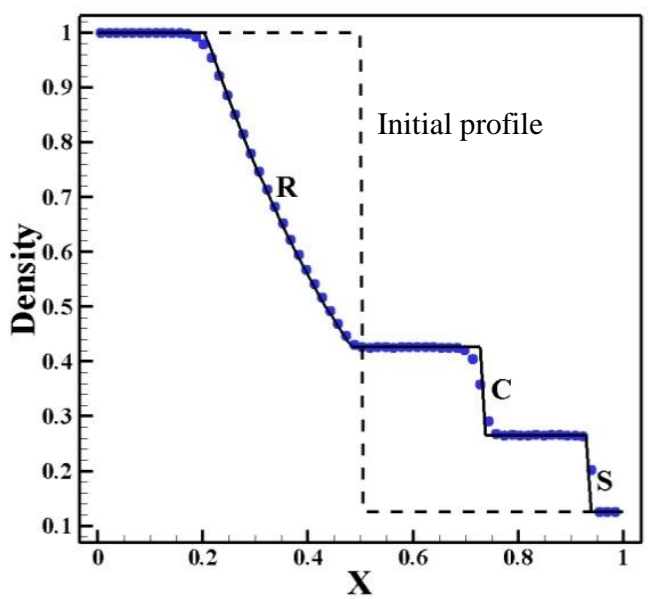

c

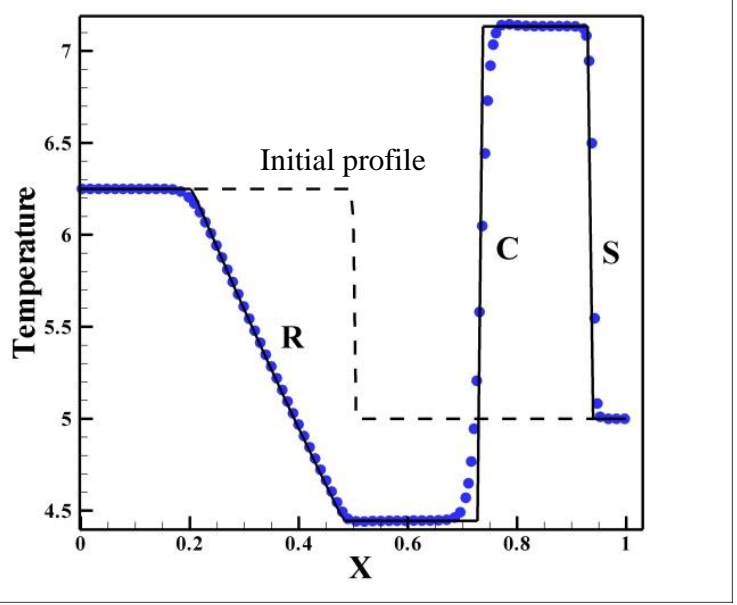

Exact Solution

3DYNAFS-COMP

Figure 1. Variation of the normalized (a) pressure, (b) density, and (c) temperature along the length of shock tube for a weak shock (initial pressure ratio of 10) at $t=0.25$. Quantities are normalized with the initial conditions (shown in dashed lines) on the high-pressure side of the tube, $X=0$. The shock wave, the rarefaction wave and the contact discontinuity are shown using the symbols $S, R$ and $C$ respectively, and are compared with the exact solution. 
a

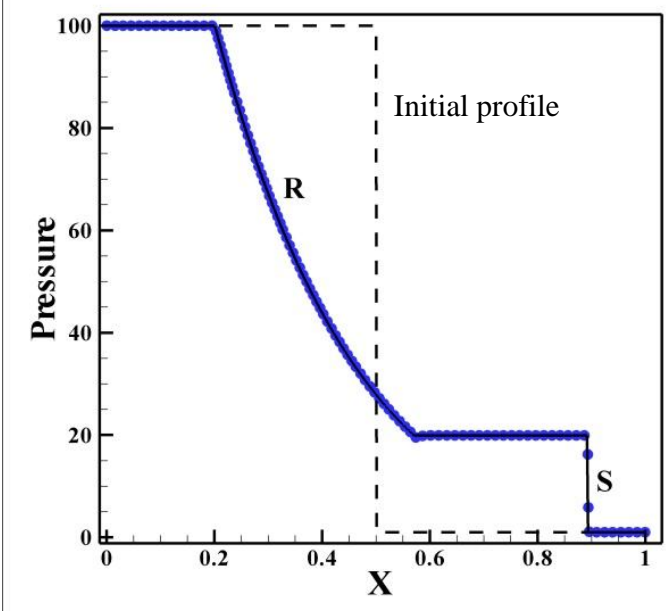

$\mathrm{b}$

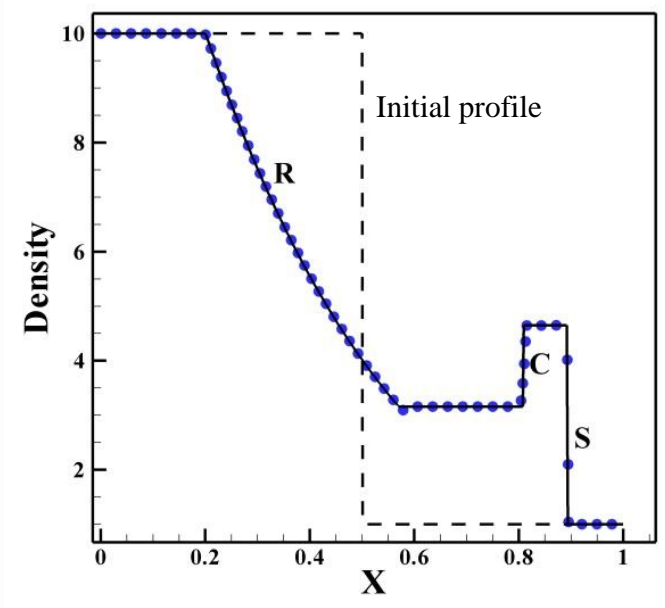

c

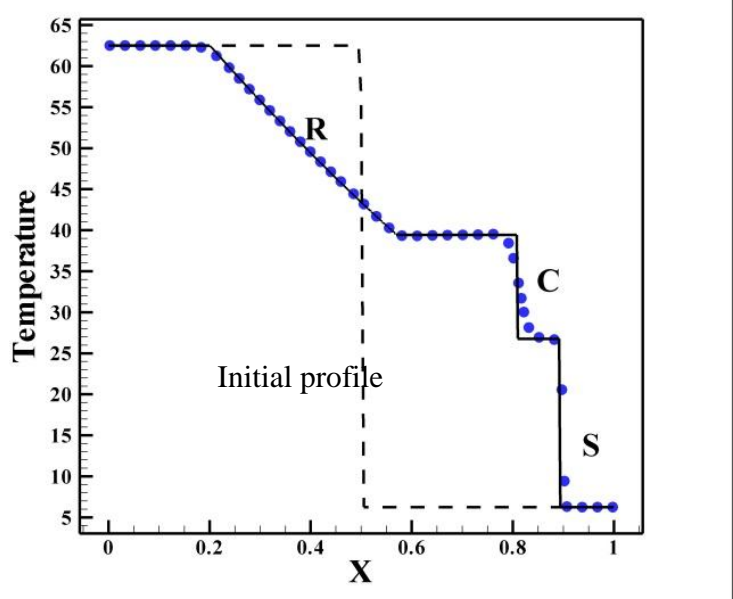

Figure 2. Variation of the normalized (a) pressure, (b) density, and (c) temperature along the length of shock tube for a strong shock (initial pressure ratio of 100) at $t=0.08$. Quantities are normalized with the initial conditions (shown in dashed lines) on the high-pressure side of the tube, $X=0$. The shock wave, the rarefaction wave and the contact discontinuity are shown using the symbols $S, R$ and $C$ respectively and are compared with the exact solution. 


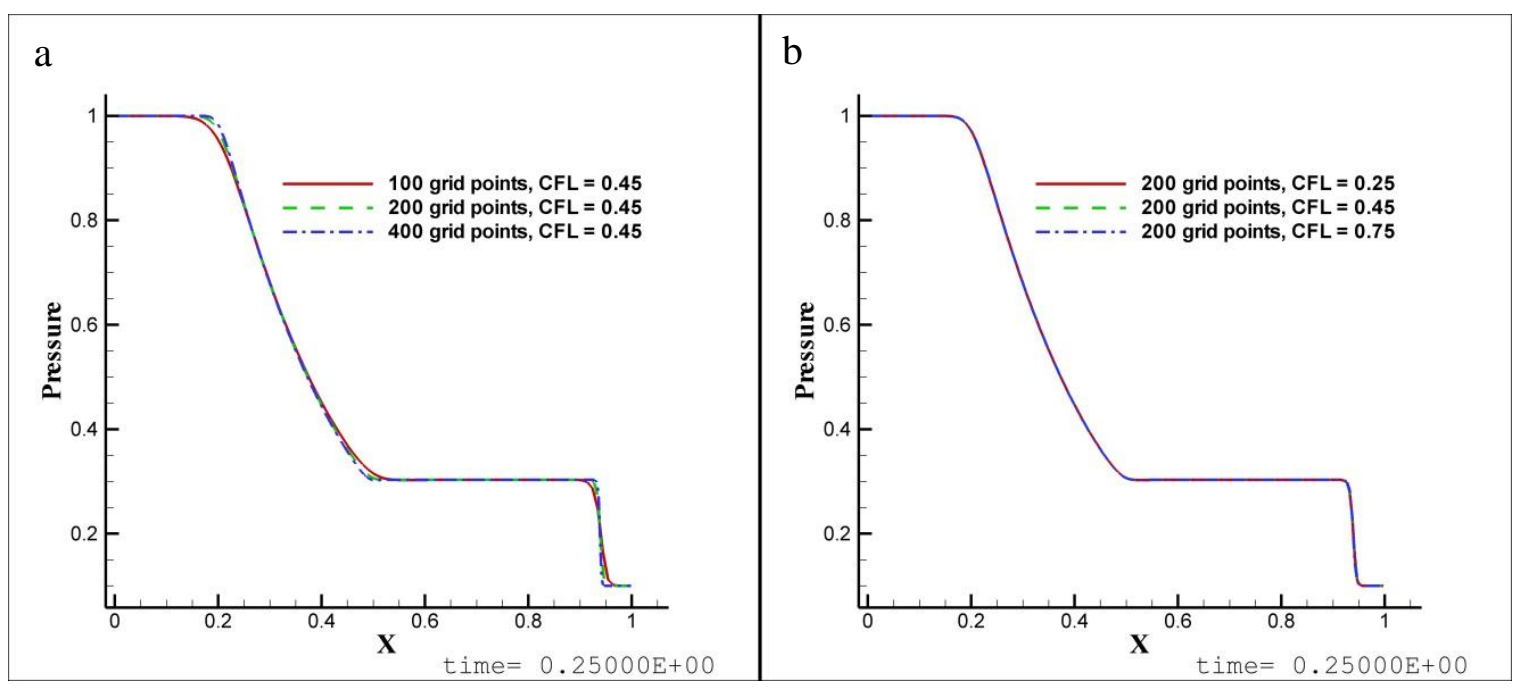

Figure 5. (a) Influence of the grid resolution on the normalized pressure along the length of the shock tube, and (b) influence of the CFL number (time step) for a space resolution of 200 grids per unit length.

\subsection{Error and convergence analysis}

This section analyses the convergence of the present multi-material solver when the grid density increases. To do so we examine the solution of Sod's shock tube solution for different grid densities. Figure 6 compares the density obtained with four increasing resolution grids with the exact solution obtained in [26]. Figure 6a shows the overall view of the solutions, while Figure $6 \mathrm{~b}$ shows a zoom around the shock location at $x \simeq 0.938$ and Figure $6 \mathrm{c}$ shows a zoom around a contact discontinuity at $x \simeq 0.731$.
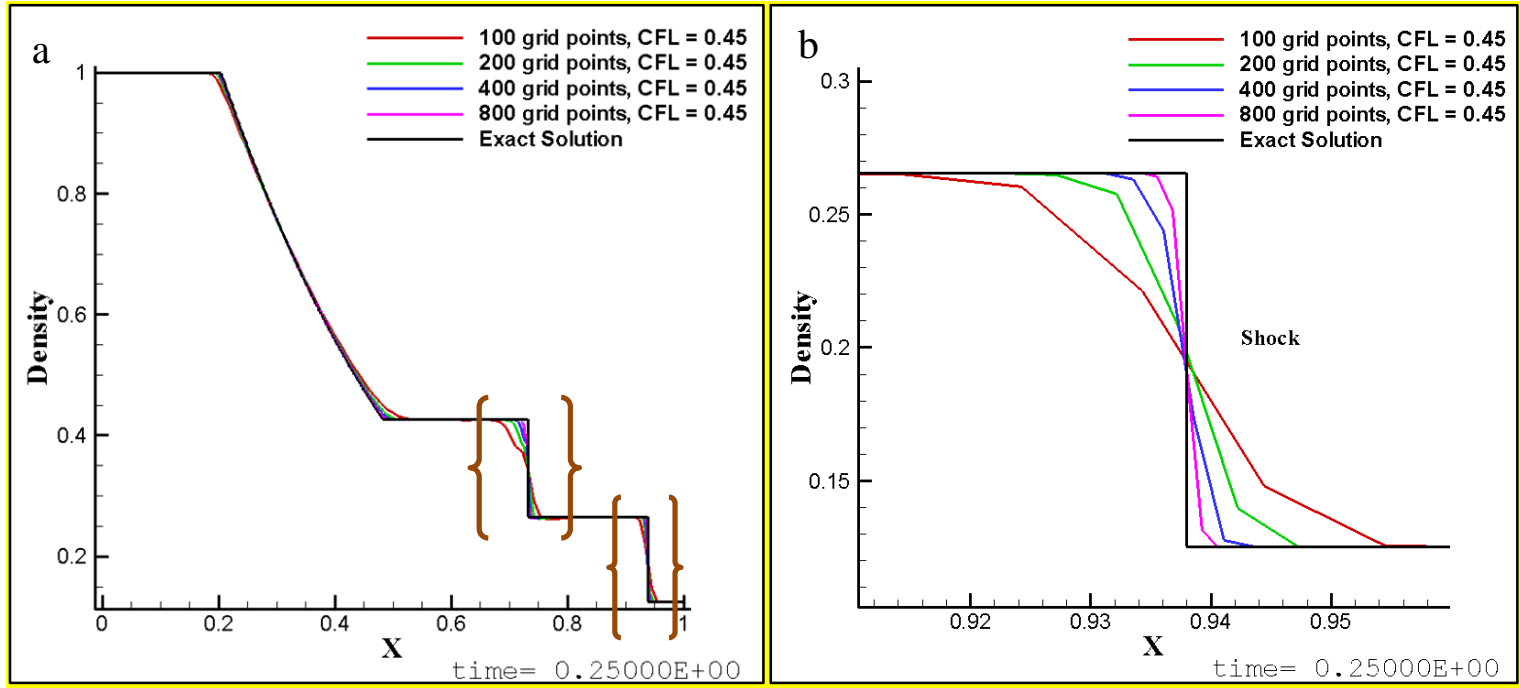


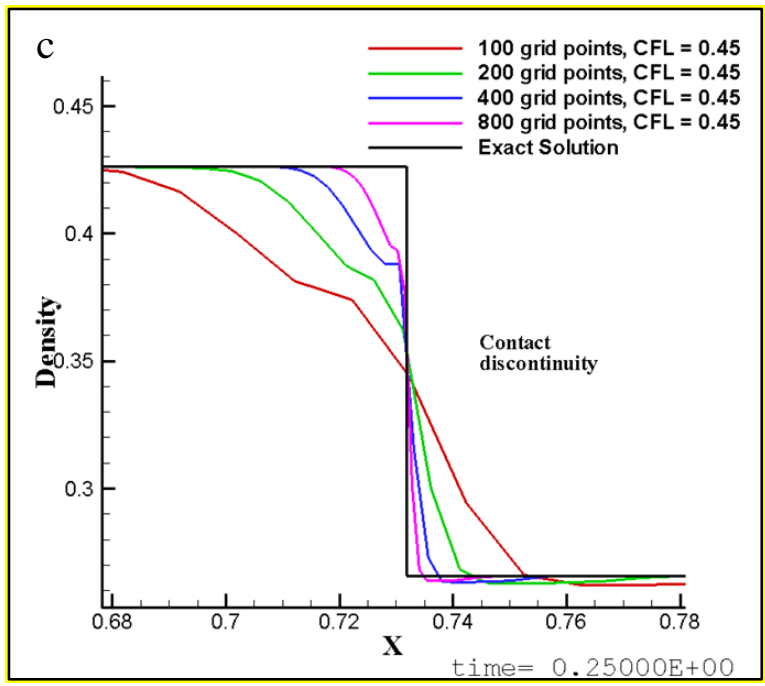

Figure 6. Influence of the grid resolution on the normalized density along the length of the shock tube. (a) Overall view of the solutions. (b) Zoom on the solution near the shock. (c) Zoom on the solution near the contact discontinuity.

The figures show that the solution converges to the exact solution as the grid is refined. To provide a quantitative evaluation we computed a measure, $\varepsilon_{d}$, of the largest error in the density, which occurs around discontinuity features such as the shock and the contact discontinuity,

$$
\varepsilon_{d}=\frac{1}{\left(x_{1}-x_{0}\right)} \int_{x_{0}}^{x_{1}} \frac{\left|\rho-\rho_{\text {exact }}\right|}{\rho_{\text {exact }}} d x \text {, }
$$

where $x_{0}$ and $x_{1}$ are selected to enclose completely the deviations from the exact solution at the shock or contact discontinuity; for instance $x_{0}=0.89$ and $x_{1}=0.97$ for the shock while $x_{0}$ $=0.65$ and $x_{1}=0.85$ for the contact discontinuity. 


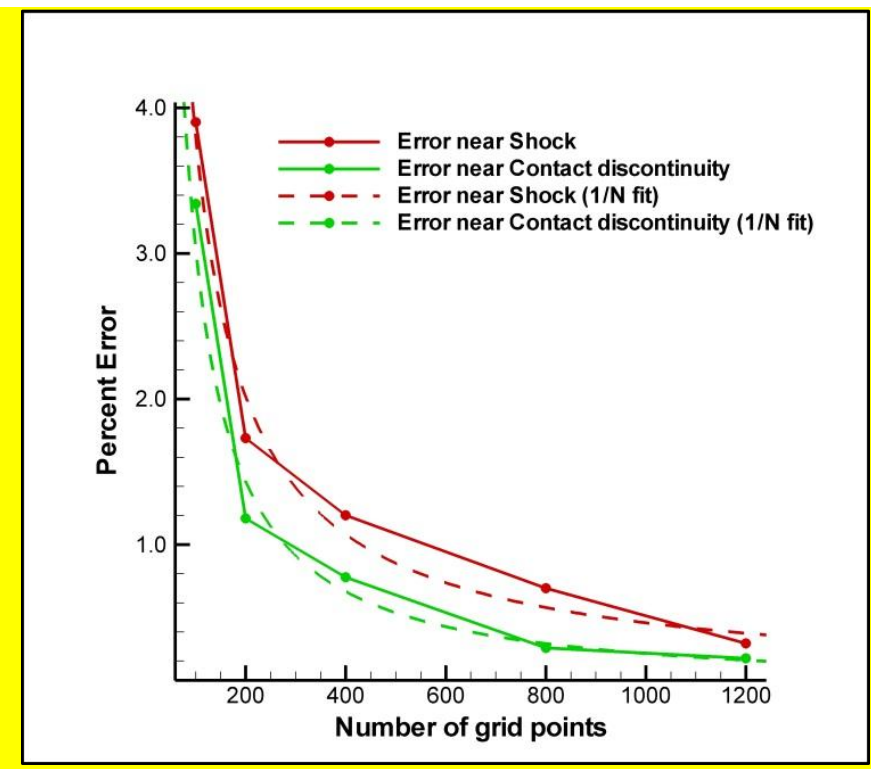

Figure 7. Percent error in the density when compared to the exact solution (see Equation 42 ) in the vicinity

of shock and contact discontinuity.

Figure 7 shows the actual error and a power fit to the error data, $N^{-\alpha}$. The value of $\alpha$ is $\sim 0.9$ for the shock and $\sim 1.08$ for the contact discontinuity.

Figure 8 also compares the temporal variation of the solution for different resolutions at the location $x=0.938$ with the exact solution. This location corresponds to the exact position of the shock at $\mathrm{t}=0.25 \mathrm{~s}$. The temporal variation of the density shows the improvement with the grid resolution in the computation of the arrival time of the shock.
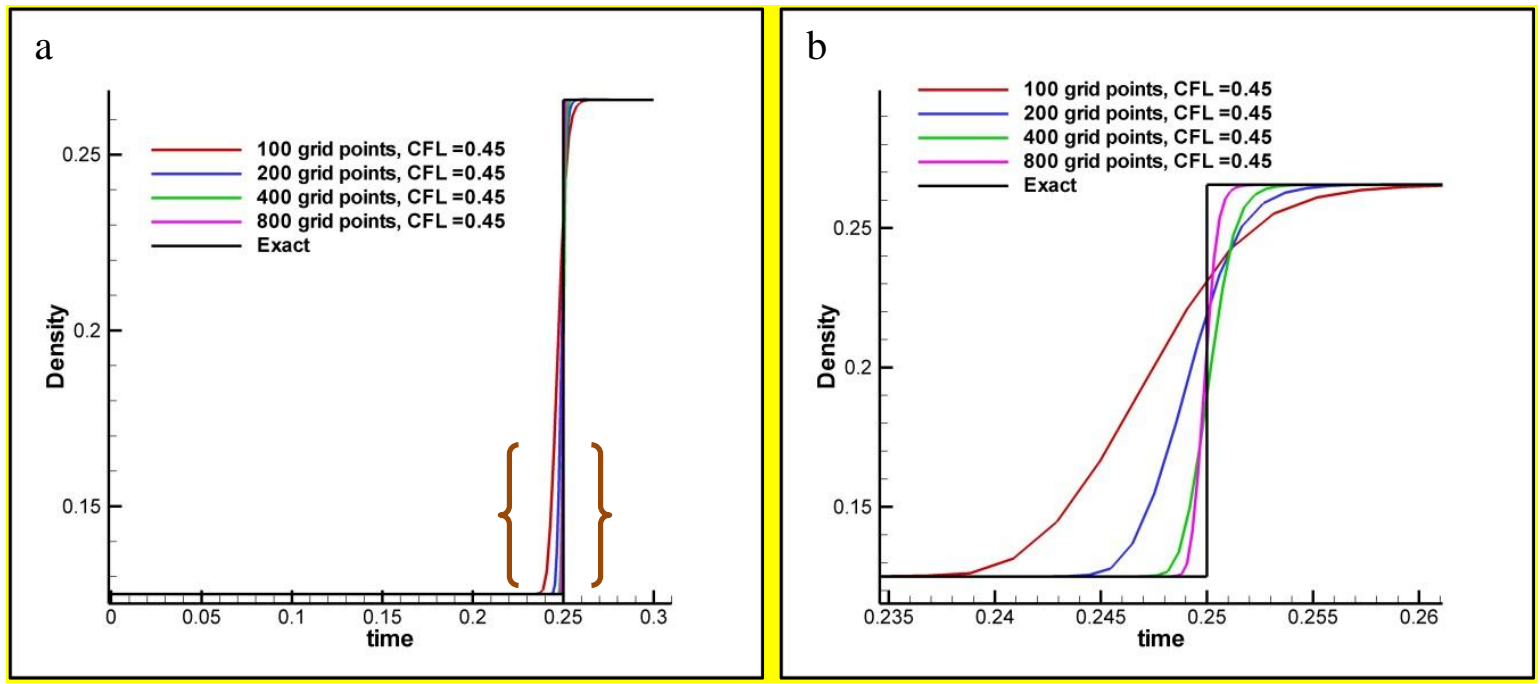
Figure 8. Influence of the grid resolution on the temporal variation of the normalized density at $x=0.938$. (a)

Full solution. (b) Zoom of the solution around $t=0.25$.

As in the previous paragraph, the error, $\varepsilon_{T}$, is computed using a time window such that the discontinuity for all the resolutions is captured and is given by:

$$
\varepsilon_{T}=\frac{1}{\left(t_{1}-t_{0}\right)} \int_{t_{0}}^{t_{1}} \frac{\left|\rho-\rho_{\text {exact }}\right|}{\rho_{\text {exact }}} d t
$$

For error computation at $x=0.938, t_{0}$ and $t_{1}$ for the case of shock are 0.23 and 0.27 . The computed error for different grid resolutions is shown in Figure 9. The figure also shows a power fit to the error data, $N^{-\beta}$, with $\beta=1.1$.

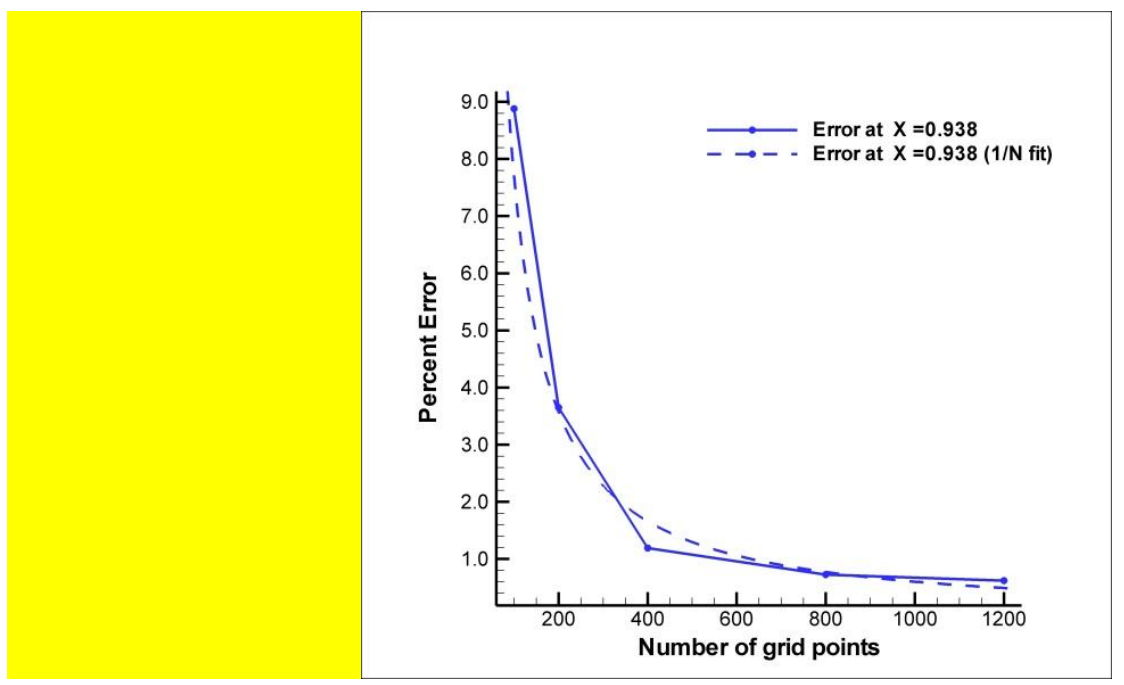

Figure 9. Percent error in the density compared to the exact solution at $x=0.938$ (see Equation 43).

Finally, we also determine the experimental order of convergence (EOC) using the following expression: 


$$
E O C=\log _{2}\left(\frac{\left\|\varepsilon_{N / 2}\right\|}{\left\|\varepsilon_{N}\right\|}\right),
$$

where $\left\|\varepsilon_{N}\right\|$ is the norm of the solution error and $N$ is the number of grid points. Here, we use the $l^{l}$ norm of the density error computed as:

$$
l^{1}=\Delta x \sum_{j=1}^{N}\left|\rho\left(x_{j}\right)-\rho_{\text {exact }}\left(x_{j}\right)\right|
$$

The computed norms and EOC are shown in Table 2. The numbers clearly show that the error reduces as the grid points increase and that the value of EOC is near one. The presence of strong discontinuities in the solution of the present problem, reduces the order of convergence to 1 near those regions.

\begin{tabular}{rrrr}
\hline Number of grid points & $\boldsymbol{l}^{l}$-Norm & \multicolumn{1}{l}{ EOC } \\
\hline 100 & $5.9 \mathrm{E}-03$ & \\
200 & $2.6 \mathrm{E}-03$ & 1.17 \\
& & \\
400 & $1.4 \mathrm{E}-03$ & 0.89 \\
800 & $6.8 \mathrm{E}-04$ & 1.04
\end{tabular}

Table 2. I' norm and EOC values computed for Sod's shock tube problem.

We also computed the EOC for a smooth solution problem given in [27]. The problem considers the exact solution of the one-dimensional Euler equation for the propagation of a sinusoidal space variation of the density :

$$
\begin{gathered}
\rho(x, t)=1.0+0.2 \sin (\pi(x-u t)), \\
u(x, t)=0.1, p(x, t)=0.5 .
\end{gathered}
$$


The solution is propagated in time while applying periodic boundary conditions. Figure 10 compares the solution for this problem at $\mathrm{t}=0.5$ for different grid resolutions with the exact solution.

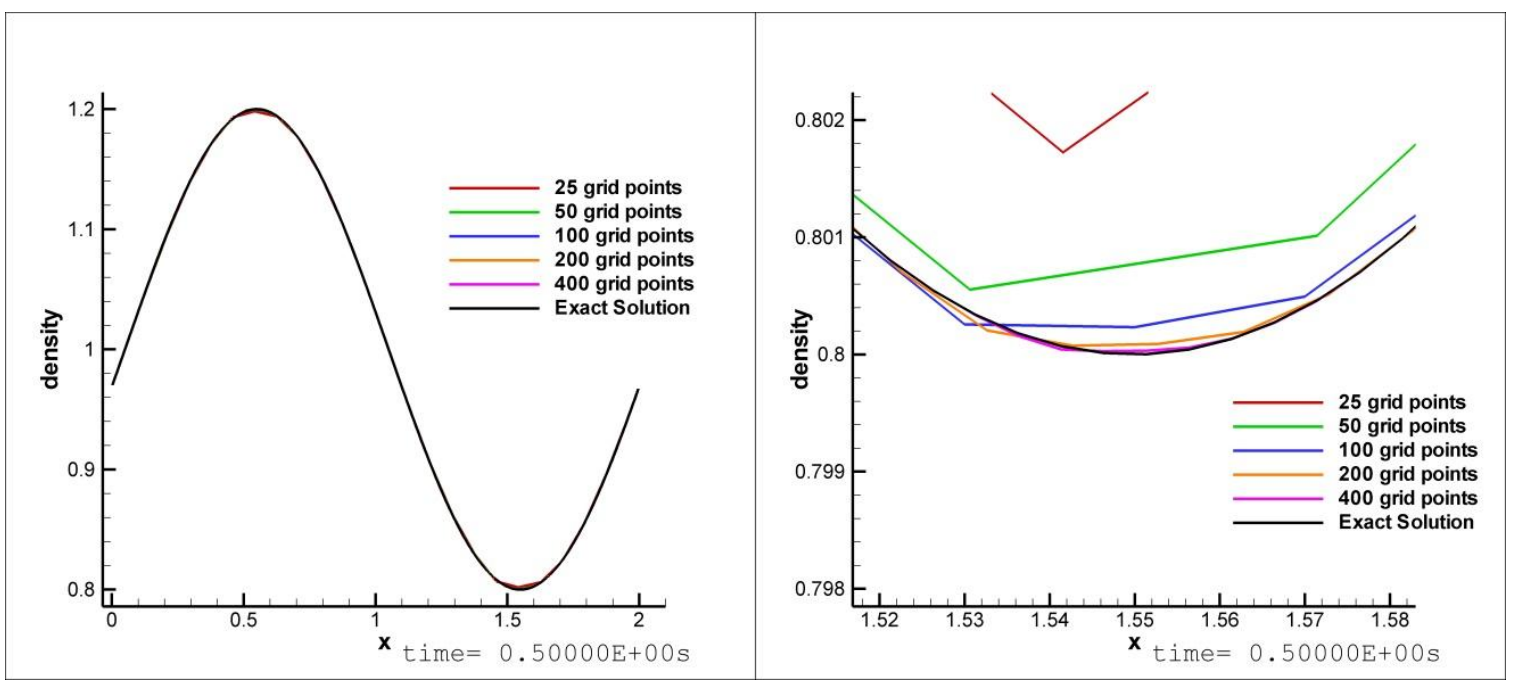

Figure 10. Influence of the grid resolution on the density solution along the length of domain for the smooth sinusoidal solution of the Euler equation as suggested in [27].

The figure also shows a zoom at the trough of the sinusoid at $(x=1.55)$ and illustrates the convergence of the solution as the number of grids is increased. The error in the solution using (42), for $x_{0}=1.0$ and $x_{1}=2.0$ for different grids is shown in Figure 11. The power fit to the error data gives $\alpha=2.19$ in this case. 


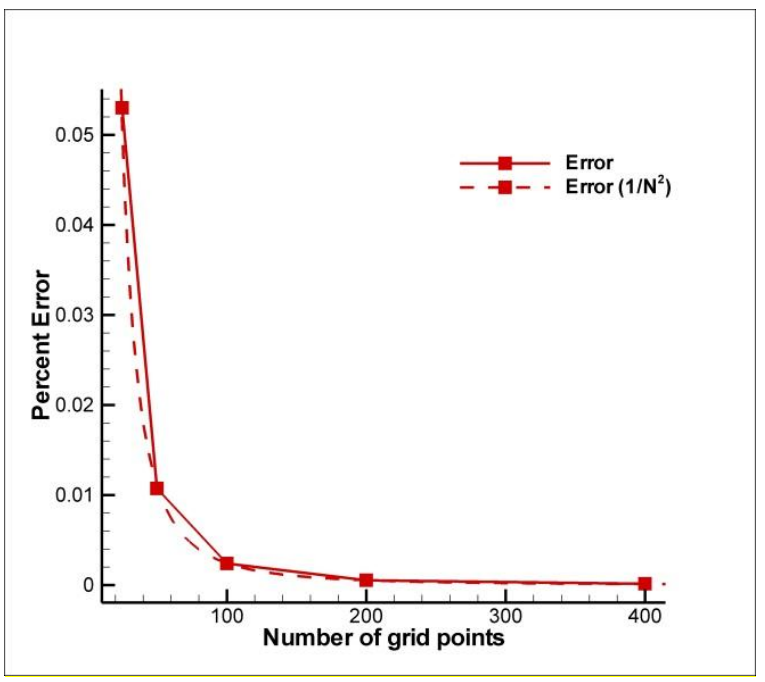

Figure 11. Percent error in the density when compared to exact solution for the smooth sinusoidal solution of the Euler equation in [47].

The EOC computed for this problem is tabulated in Table 3 and is seen to be higher than 2 indicating second order convergence in absence of discontinuity regions.

\begin{tabular}{rrrr}
\hline Number of grid points & $l^{1}$-Norm & EOC \\
\hline 25 & $5.3 \mathrm{e}-4$ & \\
\hline 50 & $1.1 \mathrm{e}-4$ & 2.30 \\
100 & $2.4 \mathrm{e}-5$ & 2.16 \\
& & & \\
200 & $5.2 \mathrm{e}-6$ & 2.21 \\
400 & $1.2 \mathrm{e}-6$ & 2.08 \\
\hline
\end{tabular}

Table 3. I'norm and EOC values computed for the smooth sinusoidal solution of the Euler equation in [47].

\subsection{Two-material shock tube problem}

Accurately modeling a two-material shock tube problem represents a stringent test case to verify the implementation of the interface treatment. This section demonstrates the approach 
for a two-material shock tube problem. The given setup has high-pressure air on one side and water at ambient condition on the other side of a discontinuity surface. The initial conditions for the problem are given in Table 4.

\begin{tabular}{|c|c|c|c|c|c|c|}
\hline \multirow{2}{*}{ Case } & \multicolumn{2}{|c|}{ Pressure (MPa) } & \multicolumn{2}{|c|}{ Density $\left(\mathrm{Kg} / \mathbf{m}^{3}\right)$} & \multicolumn{2}{|c|}{ Velocity (m/s) } \\
\cline { 2 - 7 } & Air & Water & Air & Water & Air & Water \\
\hline Two-Phase & 20 & 0.1 & 57.2 & 1000 & 0 & 0 \\
Shock Tube & & & & & & \\
\hline
\end{tabular}

Table 4: Initial conditions for the two-material shock tube problem presented here.

Complete equations of state are used for both materials with the pressure defined as a function of both the internal energy and the density. The ideal gas equation (2) is used for air and Tillotson equation (4) is used for water. As shown in Figure 12, the evolutions of the shock wave, the rarefaction wave, and the contact discontinuity are captured well and are in excellent comparison with GEMINI [23]. The figure shows the pressure, temperature, density and velocity along the tube at a selected time and are compared with GEMINI. The location of the shock wave $(X=1.626 \mathrm{~m})$, the contact discontinuity $(X=0.0554 \mathrm{~m})$, and the rarefaction wave $(X=-1.6767 \mathrm{~m})$ are represented using the symbols $\mathrm{S}, \mathrm{C}$ and $\mathrm{R}$ respectively. It should also be noted that the ratios of local air and water pressures are equal to the ratio of impedances. The pressure in the water increases to $19.39 \mathrm{MPa}$ due to the propagation of the shock wave, while the pressure in air decreases to $0.51 \mathrm{MPa}$ due to propagation of the rarefaction wave making the pressure distribution ratio decrease from 200 to 38 . The sound speeds of air and water corresponding to the initial conditions given in Table 4 are $699 \mathrm{~m} / \mathrm{s}$ and 1,483 m/s respectively. The impedance ratio of water to air calculated using these values is 37.05 and is very close to the resulting pressure ratio across the interface as expected. This problem is 
solved using 500 grid points in the X-direction to discretize a length of $1 \mathrm{~m}$. This serial run took $8 \mathrm{CPU}$ minutes on a $3.33 \mathrm{GHz}$ quad core computer.
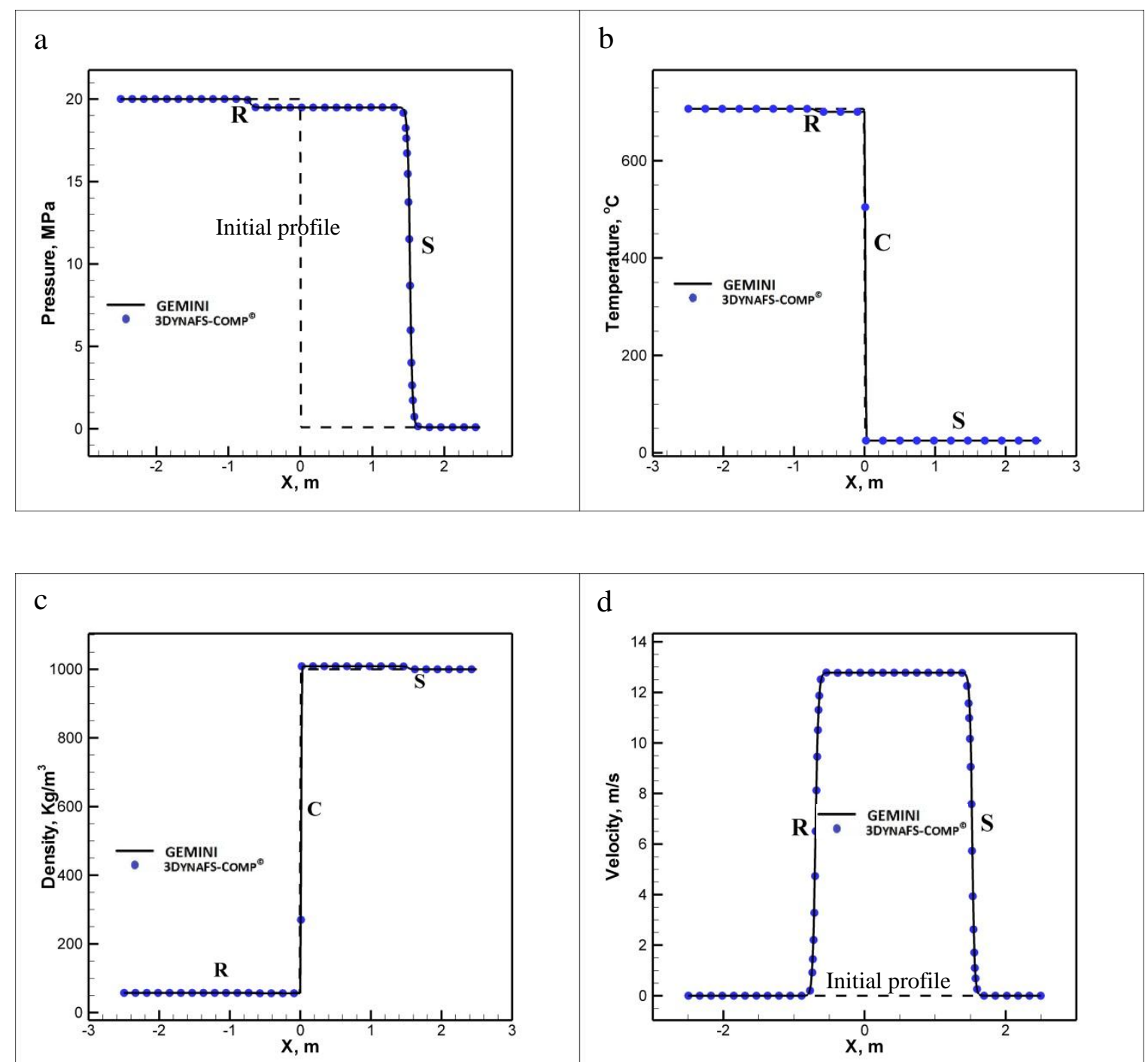

d

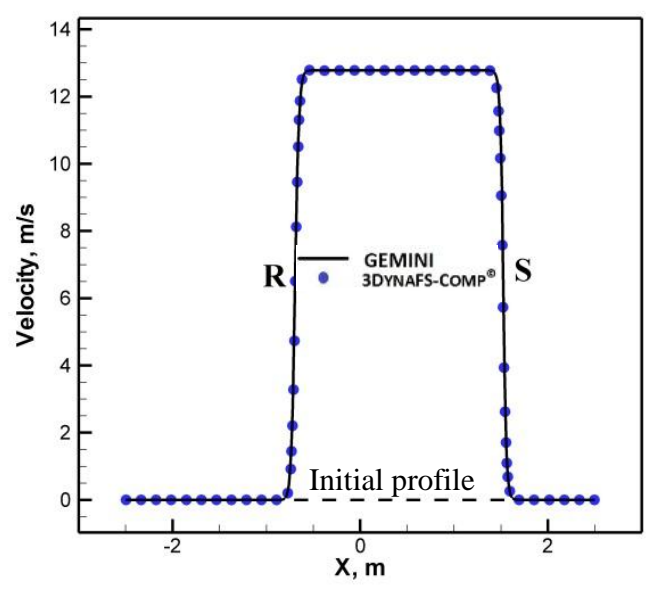

Figure 12. Variation of (a) pressure, (b) temperature, (c) density, and (d) velocity at $t=0.001$ s across the length of shock tube for a two-phase shock problem as compared with GEMINI. The initial conditions are represented using the dashed line and symbols $S, C$, and $R$ represent the location of shock wave, rarefaction wave and contact discontinuity.

Figure 13 shows the temporal variations in the temperature at two locations, one in air at $\mathrm{X}=-$ 0.2 and the other in the water at $X=0.2$. The shock wave propagation results in increase in the temperature in water while there is a decrease in the temperature of air due to propagation of 
a rarefaction wave. Figure 13 also compares the results with results from GEMINI. This example problem shows that the method presented here can handle properly shock wave propagation and interaction with an interface for a stiff pair of materials with significant difference in impedance.

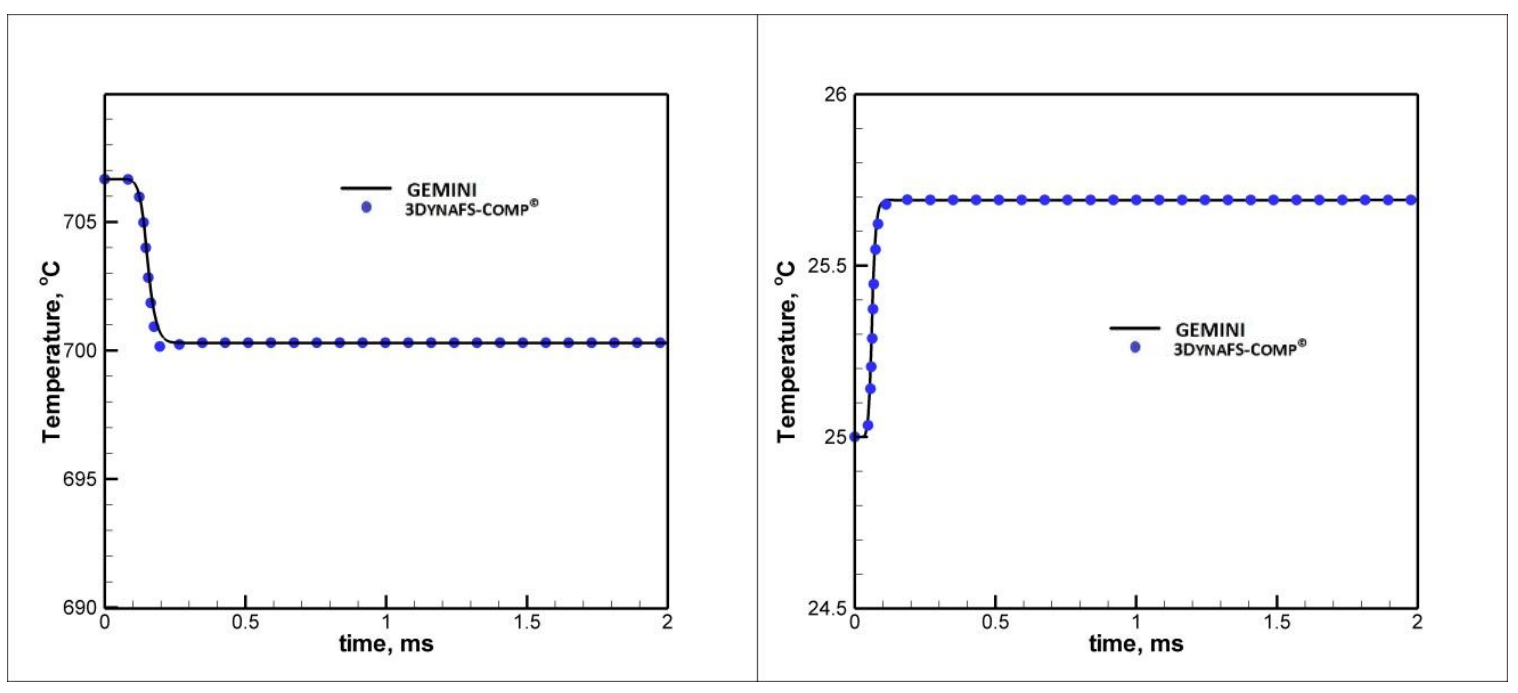

Figure 13. Temporal variation of temperature as compared to GEMINI, monitored at $X=-0.2 m$ for air (left) and $X=0.2 \mathrm{~m}$ for water (right). The total length of tube is $5 \mathrm{~m}$ spanning from $X=-2.5 \mathrm{~m}$ to $X=2.5 \mathrm{~m}$, with initial interface located at $X=0 \mathrm{~m}$. The initial pressure in air and water are $20 \mathrm{MPa}$ and $0.1 \mathrm{MPa}$ respectively. The initial temperature in air and water are $700.3^{\circ} \mathrm{C}$ and $25^{\circ} \mathrm{C}$ respectively.

\subsection{Method Verification: Artificial Multi-material Problem}

To verify the accuracy of the interface treatment of the method, we consider the following test case. We solve the Sod's Shock tube in a single material treating the two sides as two different materials (with the same properties) as in a multimaterial problem. Since the Sod's Shock tube problem has an exact solution, the comparison will evaluate the correctness of the interface treatment. This is done using the states corresponding to the weak shock problem 
given in Table 1. The problem is simulated by assigning different material numbers to corresponding states.

The comparison of the results in terms of the final density, pressure, and temperature at $\mathrm{t}=0.25$ is shown in Figure 14. The comparison clearly shows that the interface treatment is capable of capturing same results as the pure material algorithm. Small deviations in the temperature steep slope region are seen when the gridding is not fine enough, here 200 nodes along the $1 \mathrm{~m}$ tube length. These differences disappear when the number of grids is larger, here 400 nodes.
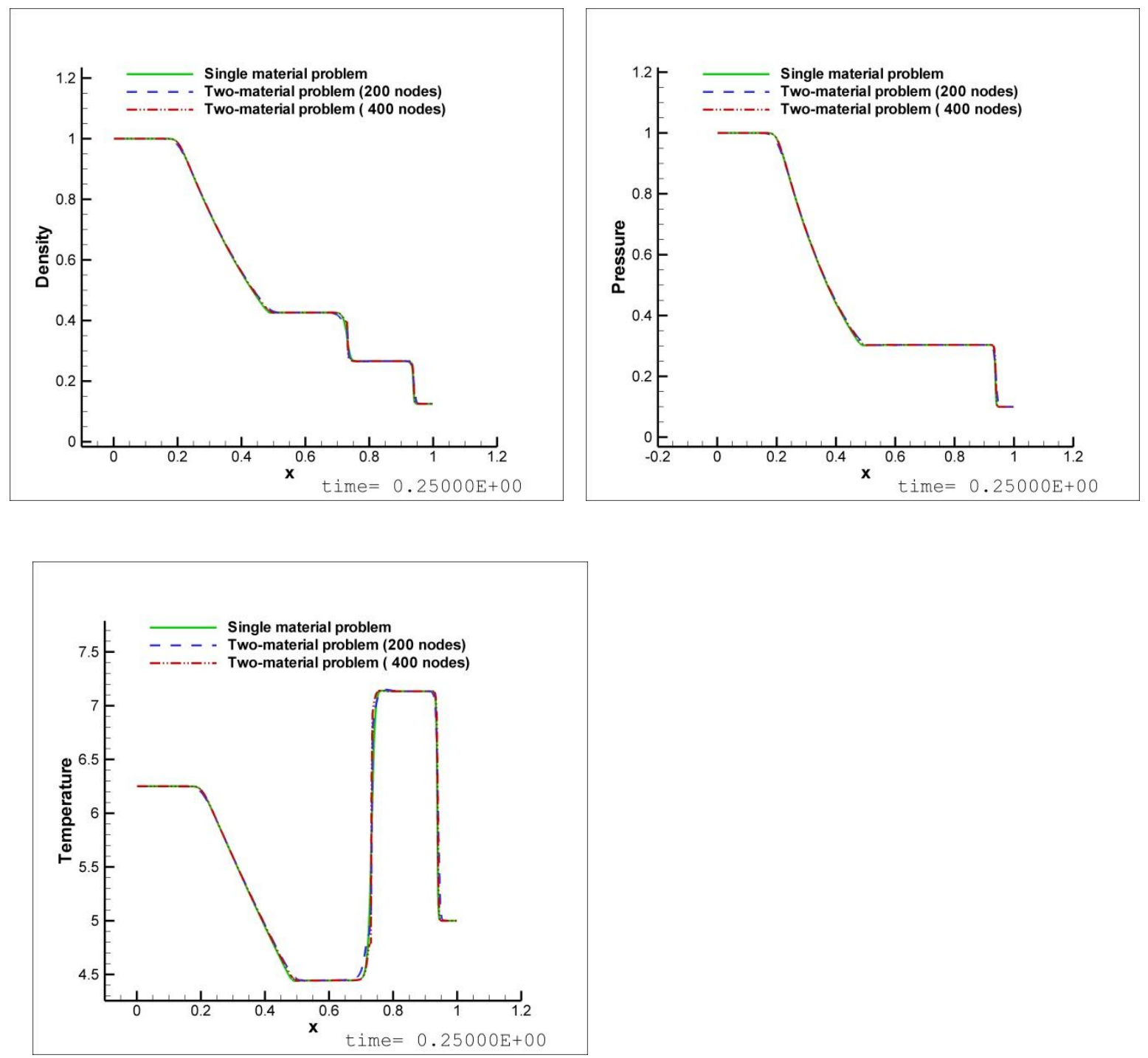
Figure 14. Comparison of Sod's shock tube solution when the material is treated as a single material problem and a two-material problem. (a) Normalized pressure, (b) density, and (c) temperature along the length of shock tube at $t=0.25$.

\subsection{Efficiency of the method}

This section examines the efficiency of the present method, which applies the remap procedure only at the interface when it is compared to a method, which uses Lagrange and remap procedure everywhere in the domain. The methods are compared using the Sod's shock tube problem. In a scenario of using the Lagrange and remap step everywhere, all the computational cells are initialized as mixed cells. The comparisons of results in terms of the final density, pressure and temperature at $\mathrm{t}=0.25$ are shown in Figure 14. Using the Lagrange and remap procedure at the interface has the advantage in reduction by $30 \%$ the computational time. Also, by using an Eulerian algorithm away from the interface, the method can use higher order discretization scheme such as MUSCL making the method both efficient and accurate. The computation was done on a $3.33 \mathrm{GHz}$, quad core machine. In this case we used 200 discrete points along the 1m tube length. 

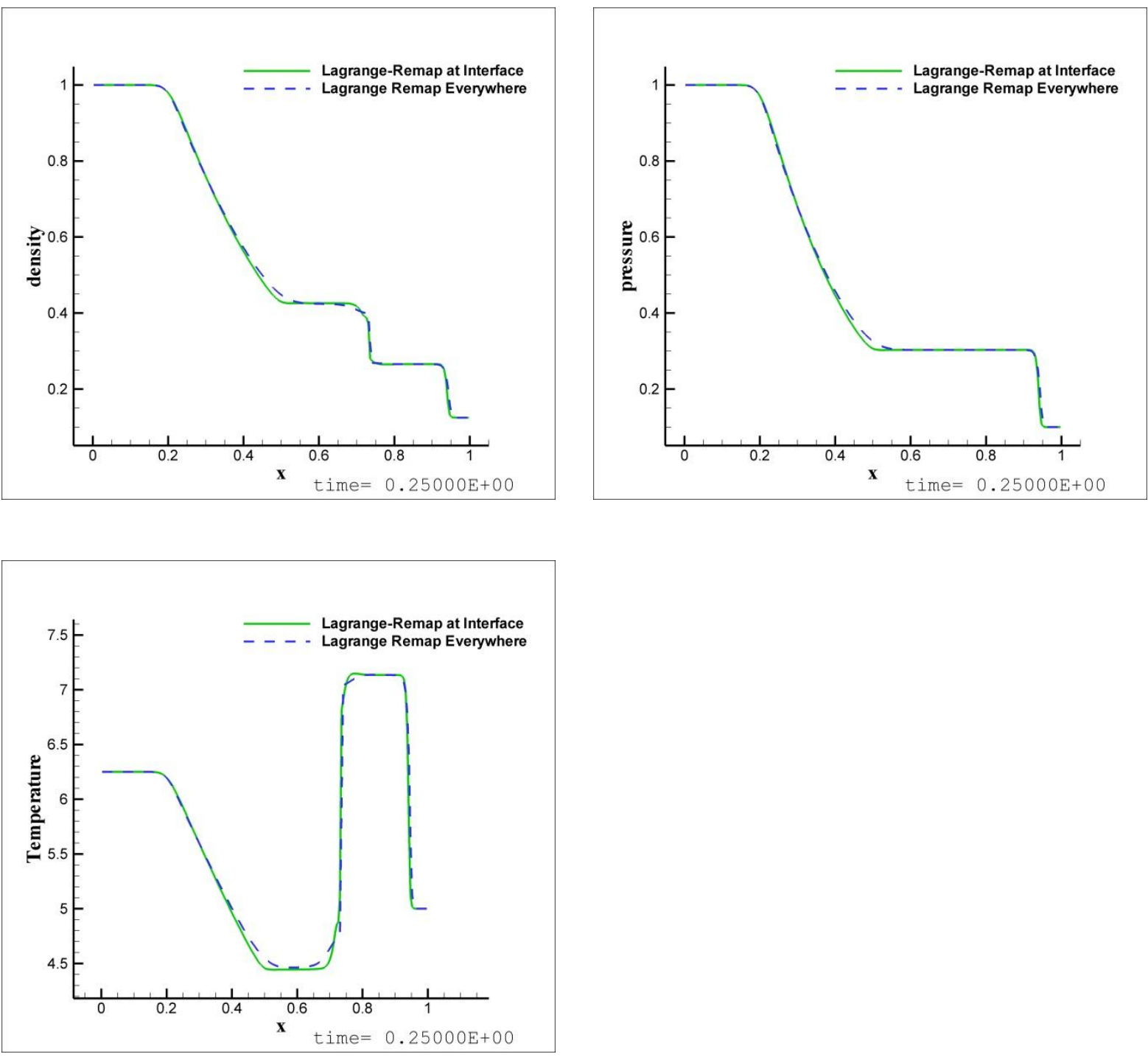

Figure 15. Comparison of Sod's shock tube solution when using the Lagrange and remap procedure everywhere versus only at the interface. (a) Normalized pressure, (b) density, and (c) temperature along the length of shock tube at $t=0.25$.

\subsection{Two-dimensional Riemann problem}

Two-dimensional Riemann problems [1] are a set of nineteen well established initial pressure, velocity, and density configurations of different fluids, resulting in combinations of non-linear waves such as shock waves, rarefaction waves, and contact discontinuities. These problems, though not possible to solve analytically, have numerical solutions, which are used to benchmark a compressible flow solver. In this work, the solution of one of such 
configurations (\# 13) is illustrated in Figure 16 and is seen to capture the wave physics well. The initial conditions for configuration \#13 are prescribed constant values of the prime variables in four quadrants of a two-dimensional domain. These conditions in nondimensional form are given in Table 5.

\begin{tabular}{|c|c|c|}
\hline & $X<0.5$ & $X>0.5$ \\
\hline$Y<0.5$ & $\begin{array}{c}\text { Quadrant I } \\
P_{0}=1.0, \\
\rho_{0}=2.0, \\
u_{0}=0.0, v_{0}=0.3\end{array}$ & $\begin{array}{c}\text { Quadrant II } \\
P_{0}=1.0, \\
\rho_{0}=1.0, \\
u_{0}=0.0, v_{0}=-0.3\end{array}$ \\
\hline$Y>0.5$ & $\begin{array}{c}\text { Quadrant III } \\
P_{0}=0.4, \\
\rho_{0}=1.0625, \\
u_{0}=0.0, v_{0}=0.8145\end{array}$ & $\begin{array}{c}\text { Quadrant IV } \\
P_{0}=0.4, \\
\rho_{0}=0.5313, \\
u_{0}=0.0, v_{0}=0.4276\end{array}$ \\
\hline
\end{tabular}

Table 5. Initial conditions for a two-dimensional Riemann problem (configuration \#13 in[1]).

The density contours in the two-dimensional domain at the non-dimensional time, $t=0.3$, are shown in Figure 16. These contours are compared with those obtained by Lax et al. [1] and are in good agreement. This problem is solved using a 400×400 grid, which is the same grid density as the one used in Lax et al. 

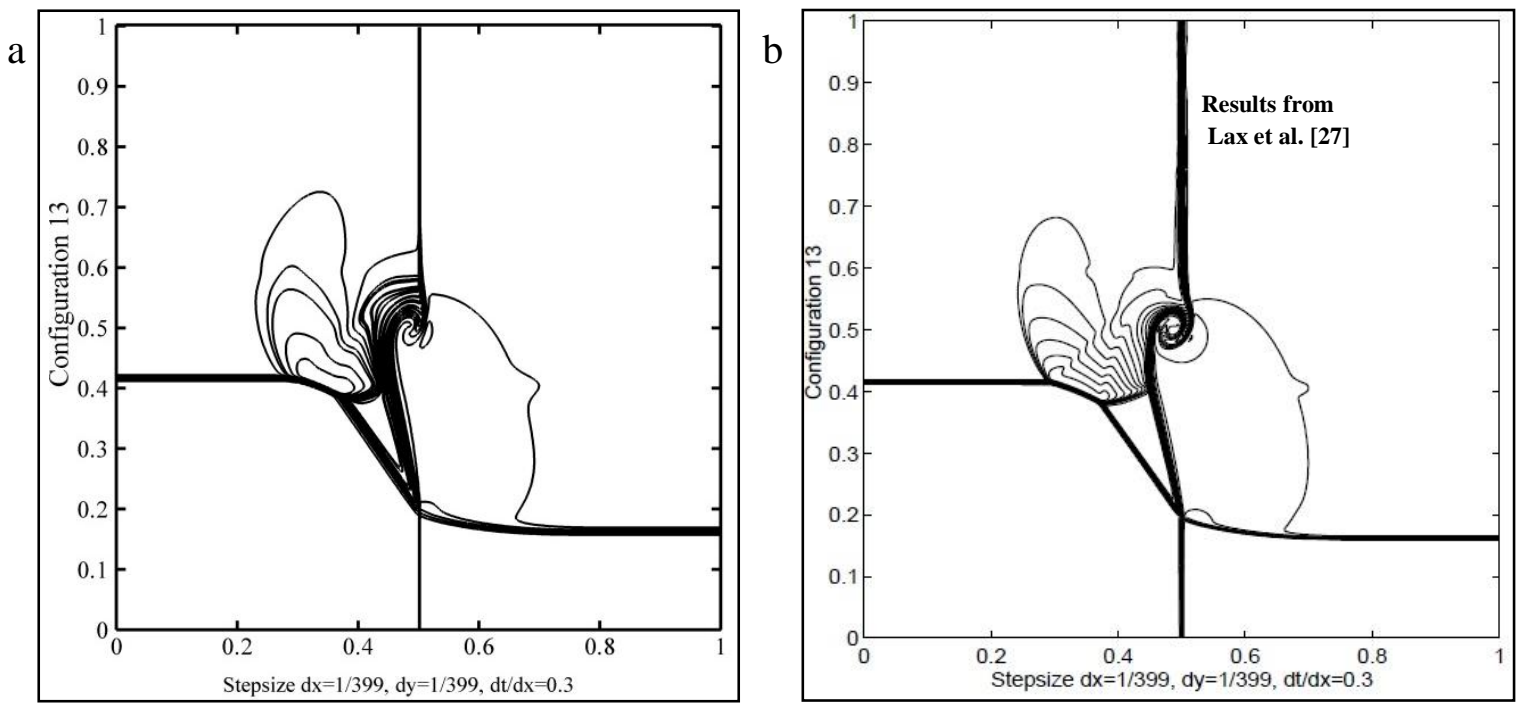

Figure 3. Density contours at non-dimensional time, $t=0.3$ for the two-dimensional Riemann problem (Configuration \#13 in [1]). (a) 3DYNAFS-COMP ${ }^{\oplus}$ results, (b) Lax et al. results in 1. The initial conditions corresponding to this problem are given in Table 5.

\subsection{Exploding Bubble}

In this section we illustrate the capability of 3 DYNAFS-COMP ${ }^{\odot}$ to treat a compressible flow with a moving free surface boundary. The problem addressed is that of the dynamics of an initially spherical bubble containing gas at a higher pressure than the surrounding infinite medium. For the example shown here, the computational domain is defined in a cubical domain of dimensions given by $X_{\max }=Y_{\max }=Z_{\max }=-5,000 \mu \mathrm{m}$ and $X_{\min }=Y_{\min }=Z_{\min }=5,000 \mu \mathrm{m}$. The bubble center is at $X=Y=Z=0$, its initial radius is $250 \mu \mathrm{m}$, its internal pressure is 5 bar, and it is surrounded by water at a pressure of 1 bar. A uniform grid density of $20 \mu \mathrm{m}$ is used to resolve the region around the bubble and a stretched grid with a geometric expansion factor of 1.2 is used away from the bubble, i.e. for $|\mathrm{X}|,|\mathrm{Y}|,|\mathrm{Z}|>500 \mu \mathrm{m}$.

$$
\Delta x_{i+1}^{n+1}=1.2 * \Delta x_{i}^{n}
$$

where, $\Delta x_{i}^{0}$ is $20 \mu \mathrm{m}$. All the domain boundaries are prescribed with non-reflective (Neumann) boundary conditions. A stretched grid is used to minimize the number of grids, while ensuring that the domain is large enough to avoid reflections from the boundaries. The initial setup of the problem is shown in Figure 17. 


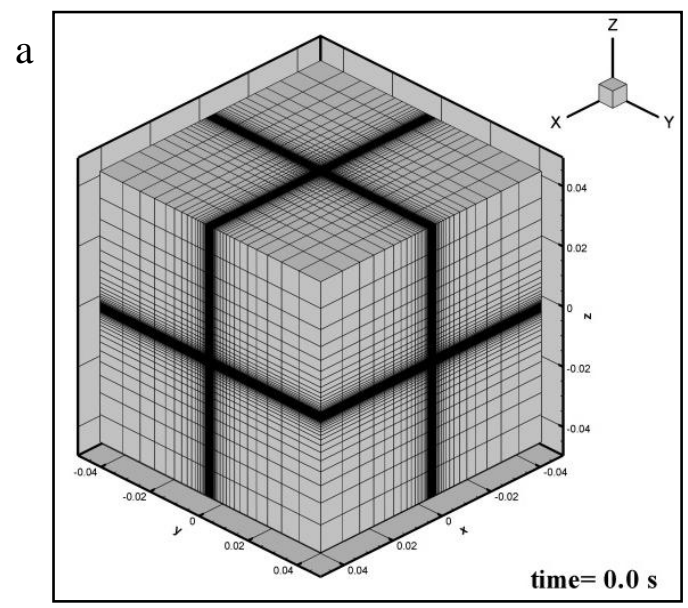

$\mathrm{b}$

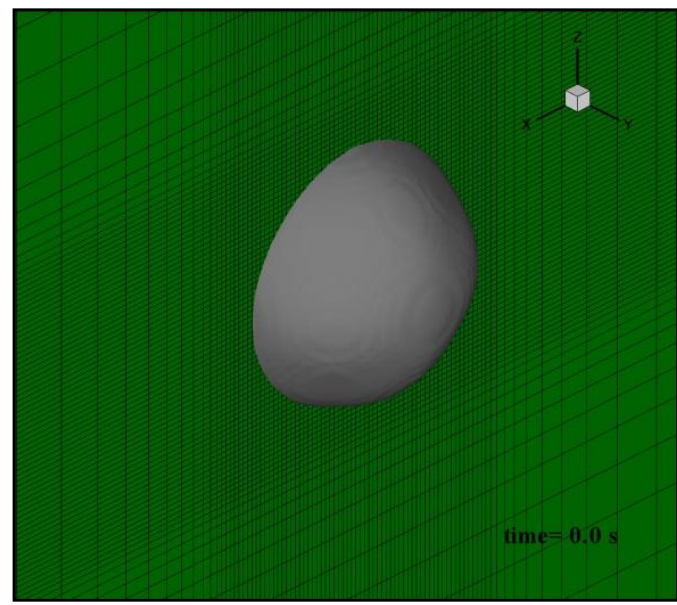

Figure 4. Initial setup for a spherical bubble initial radius $250 \mu \mathrm{m}$ in a cubical domain of $10 \mathrm{~mm}$ sides. (a) Stretched grid used for the computation (b) Initial air-water interface.

The initial bubble air pressure is 5 bars, while the pressure in the surrounding water is 1 bar.

As the air in the bubble is initially at a higher pressure than the surrounding water, releasing the bubble interface sends a high pressure wave into the surrounding medium (similar to the above shock tube problem but here in a spherical geometry). This is illustrated in Figure 18 showing, at $6 \mu$ s after release of the interface, the variation of the pressure with the radial distance from the center of the bubble. This is measured in the XOY plane along three different directions: parallel to the $\mathrm{X}$-axis, parallel to the Y-axis, and along a diagonal. Figure 18 clearly shows that the variation in pressure along multiple radial directions is almost the same. 


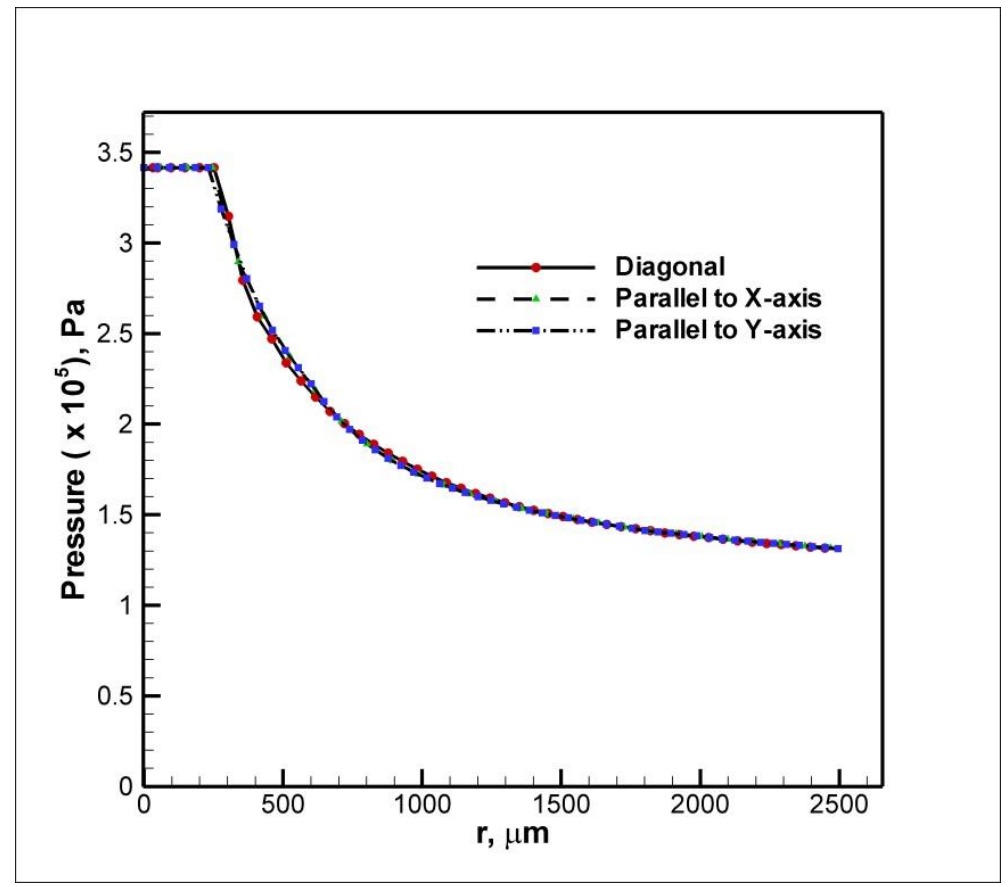

Figure 5. Variation at $t=6 \mu$ s of the shock front pressure measured in the XOY plane along the radial direction in three different directions. Air bubble of initial size $250 \mu \mathrm{m}$ and initial inner pressure 5 bar released in water where the initial pressure is 1 bar.
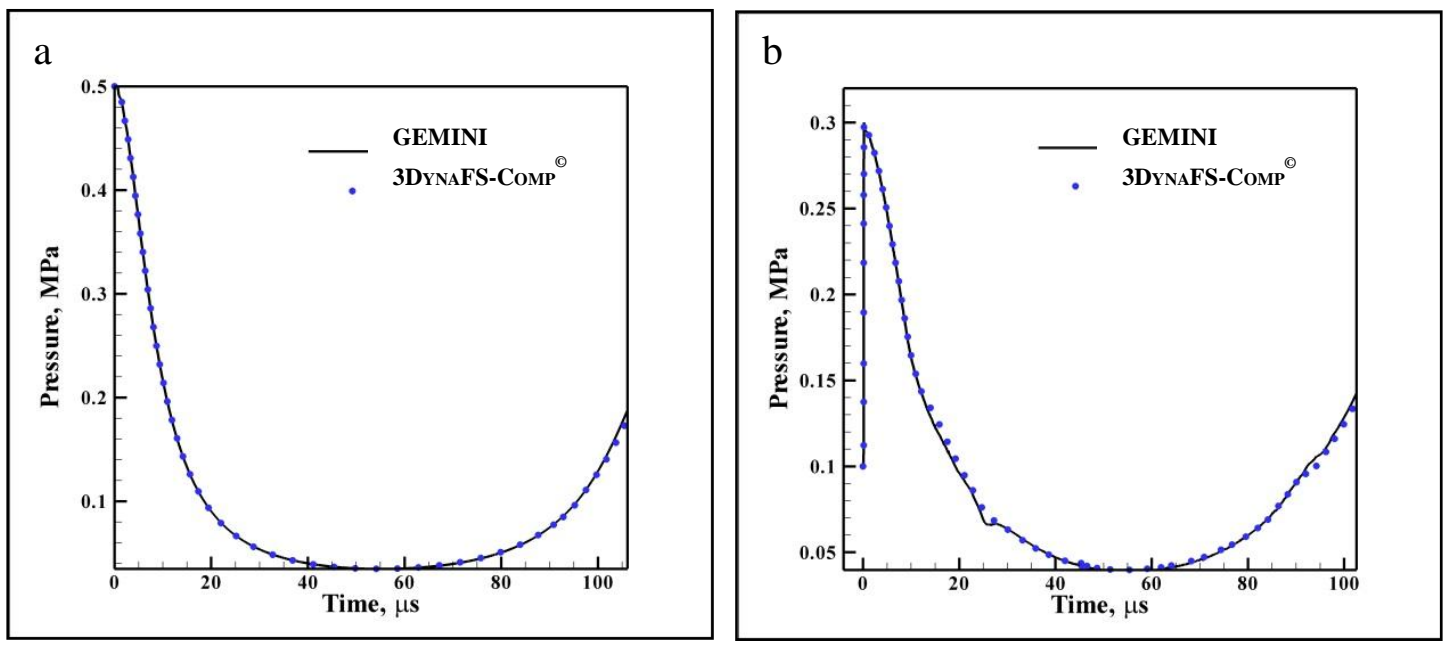

Figure 6. Comparison of the bubble and liquid pressure versus time obtained by 3DYNAFS-COMP ${ }^{(}$and GEMINI. (a) Pressure in the gas at the center of the bubble $(0,0,0)$ and (b) pressure in the liquid at $(0,0,300 \mu \mathrm{m})$. Initial conditions: air bubble radius $250 \mu \mathrm{m}$, air pressure 5 bar released in an infinite water medium where the initial pressure is 1 bar.

This outward interface motion results in a decrease in the gas pressure inside the bubble (see Figure 19(a)) and increase in bubble volume (Figure 20). This expansion phase continues 
until the bubble reaches its maximum radius (minimum gas pressure) and eventually collapses. The pressure is monitored at two locations, one at the center of bubble and another outside the bubble at the location $(0,0,300 \mu \mathrm{m})$. The temporal variations of the pressure at these two locations (Figure 19) is in excellent agreement with GEMINI [23].

The evolution of the bubble equivalent radius (sphere with the same volume) compared for the first cycle with that computed by GEMINI is shown in Figure 20 (a). The figure clearly demonstrates that the two codes are in good agreement and the developed framework has the desired capabilities to capture bubble dynamics. The decrease in bubble pressure with increase in bubble volume during the first bubble period is shown in Figure 20 (b). The plot clearly captures the physics of the problem with the bubble maximum volume matching with the minimum air pressure and with the bubble volume minima matching with the pressure maxima.

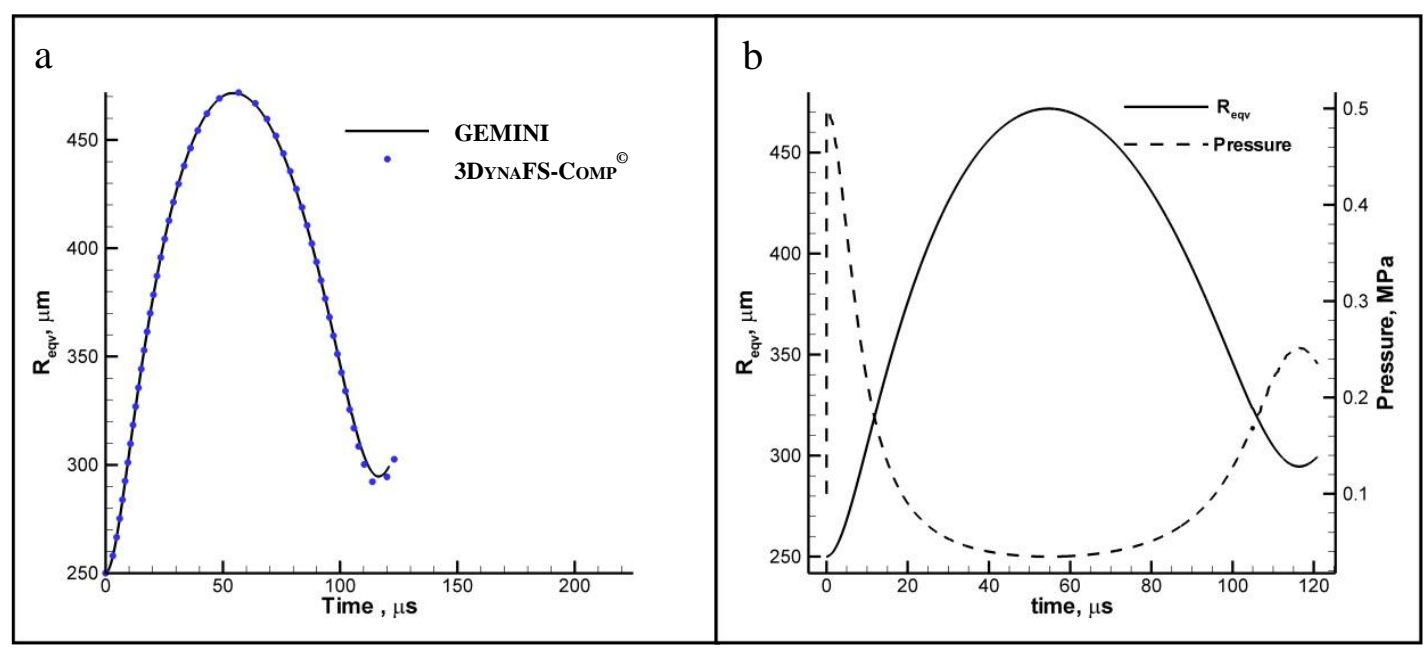

Figure 7. (a) Comparison of the time evolution of the bubble equivalent radius based on the bubble volume between 3DYNAFS-COMP ${ }^{\odot}$ and GEMINI. (b) Corresponding temporal variation of the gas pressure at the center of the bubble along the equivalent bubble radius. Air bubble initial size $250 \mu \mathrm{m}$ and initial pressure 5 bar released in an infinite medium where the initial pressure is 1 bar.

\subsection{Shock wave interaction with a cylindrical bubble}


This section describes a high-resolution computation of the collapse of an initially cylindrical bubble of radius $0.5 \mathrm{~mm}$ when impacted by a shock wave carrying a high-pressure loading of 1GPa. Similar to the examples shown above, this problem is of interest to many fields, e.g. biomedical industry, air force, and naval laboratories. The initial setup of the problem has an air bubble of internal pressure of 1 atm. at equilibrium with the surrounding water also at $1 \mathrm{~atm}$. The setting of the problem and the gridding used are shown in Figure 21.

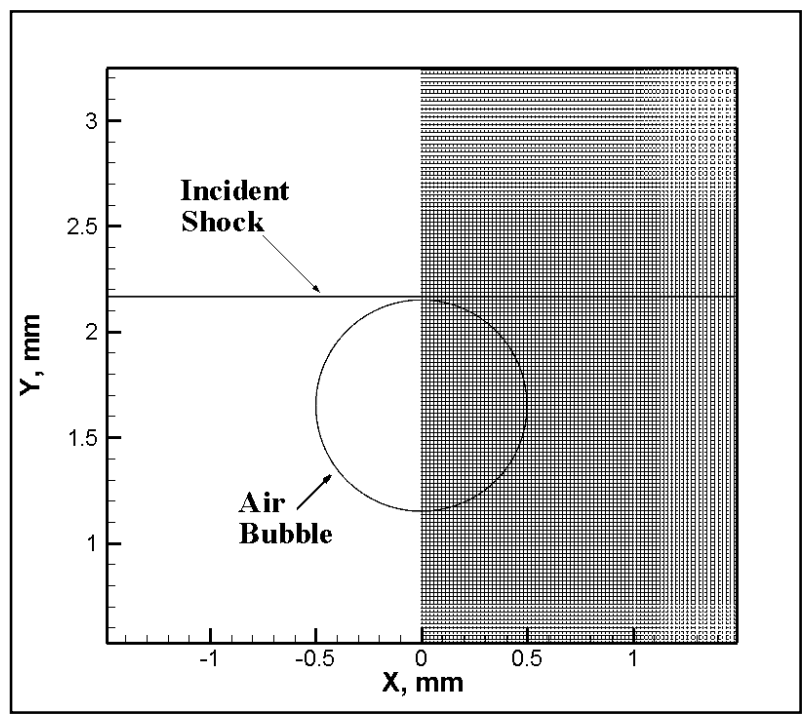

Figure 8. Interaction of a high-pressure incident shock wave (1 GPa) with an air bubble of radius $0.5 \mathrm{~mm}$ at ambient conditions. The left panel shows the setup and half the computational domain. The right panel shows the $434 \times 784$ stretched grid used to obtain high resolution simulations with $250 \times 250$ grid points to resolve the bubble radius.

A high resolution $434 \times 784$ stretched grid is used with the bubble circular cross-section comprising 250×250 grid points (Figure 21). This high-resolution grid enables to capture the physics of the problem including bubble breakup as shown in Figure 22. The various instances leading to the final collapse of the bubble are highlighted using Schlieren-like relative density variations [35]. These images accentuate the various features such as shock reflection using density gradients. The formula used to compute the Schlieren function, $v$, which is graphically represented, [35] is 


$$
v=0.8 * \exp \left(-\mu \frac{|\nabla \rho|}{|\nabla \rho|_{\max }}\right)
$$

where $\rho$ is the density, $|\nabla \rho|$ the magnitude of the density gradient, and $\mu$ is a parameter to adjust the contour levels or the color of contour images. A larger value of $\mu$ gives a darker image and accentuates the weak features. In this work, a value of 100 is used for $\mu$. The first captured instances in Figure 22 show a high-pressure shock wave in water impacting the bubble surface and resulting in both reflection of an expansion wave back into in water and transmission of the pressure wave into the air in the bubble. This is shown in Figure 22(a) and Figure 22(b). The incident shock wave after being diffracted by the bubble interface propagates in water while the transmitted shock wave propagates in air inside the bubble. As the speed of sound is larger in water than in air, the diffracted water wave moves much faster than the transmitted air wave. This can be clearly seen in Figure 22(b). The transmitted shock wave interacts with the bubble liquid interface resulting in multiple reflections illustrated in Figure 22(c) and Figure 22(d). In addition, the higher and earlier pressures impacting the top of the bubble result in shape deformation and development of a reentrant jet into the bubble from its top surface. This is illustrated further by the velocity vectors in Figure 22(d). As the air bubble deforms further, the distal surface of the bubble penetrates in the bubble as a reentrant jet and impacts the front surface, resulting in bubble fission and splitting into two side bubbles in Figure 22(e). Finally, the bubble disintegrates leading to tiny fragments as shown in Figure 22(f).

This exercise demonstrates the robustness of 3 DYNAFS-COMP $^{\odot}$ enabling it to capture events including total disintegration of the interface in a fully conservative framework. The results obtained in this simulation compare well with results from Hawker et al. [36] for the same problem but with different equations of state (We do not know unfortunately the equation of 
state used in [36]). Both are in good agreement and capture essential events during shock bubble interaction.

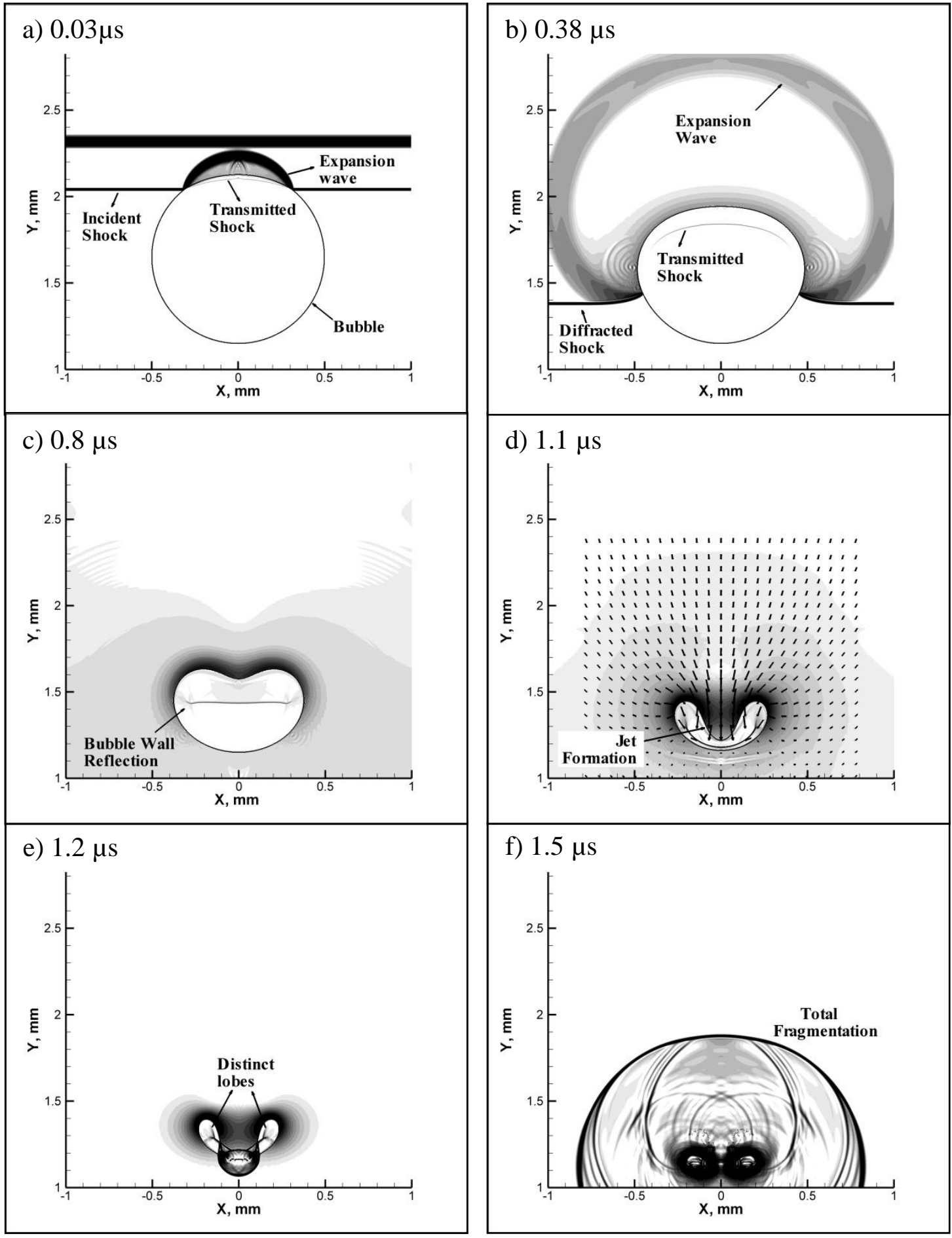

Figure 9. Interaction of a high-pressure incident shock wave (1 GPa) with an air bubble of radius

$0.5 \mathrm{~mm}$ at ambient conditions. Evolution of the bubble with time: (a) Initial shock-bubble interactions $(0.03 \mu \mathrm{s})$. (b) Transmitted shock in air and diffracted expansion wave in water $(0.38 \mu \mathrm{s})$. (c) Reflection from the air-water interface and initiation of a reentrant jet from the upper wall of the bubble $(0.8 \mu \mathrm{s})$. (d) Significant advancement of the reentrant jet (1.1 $\mu \mathrm{s})$. (e) Advancement of the jet through the lower bubble wall and bubble fission into two bubbles (1.2 $\mu \mathrm{s})$. (f) Total fragmentation of bubble (1.5 $\mu \mathrm{s})$. 


\subsection{Shock wave interaction with an initially spherical bubble}

The next example shows a shock induced collapse of an initially spherical bubble near a rigid wall. Numerous applications involve this phenomenon varying from dynamics of heterogeneous energetic materials [37] to underwater explosions and structures protection [38], to shock wave lithotripsy[ 39]. A three-dimensional cubic domain with dimensions given by $X_{\min }=Y_{\min }=Z_{\min }=-1,500 \mu \mathrm{m}$ and $X_{\max }=Y_{\max }=Z_{\max }=1,500 \mu \mathrm{m}$ is used for this problem. To initiate the problem we consider a shock front in water separating a high-pressure region (35 $\mathrm{MPa})$ from a low-pressure region at atmospheric pressure $(0.1 \mathrm{MPa})$ initially located at $\mathrm{X}=0$. A bubble of radius $250 \mu \mathrm{m}$ and initial internal pressure of $0.1 \mathrm{MPa}$ is located at a distance from the wall equal to $500 \mu \mathrm{m}$. A uniform grid density of $20 \mu \mathrm{m}$ is used throughout the domain making the total number of grid points for the problem as $3.375 \times 10^{6}$ grids. The wall boundary is prescribed using a reflective boundary condition while all other boundaries are prescribed with non-reflective boundary conditions. The schematic of the initial problem geometry is shown in Figure 23. 


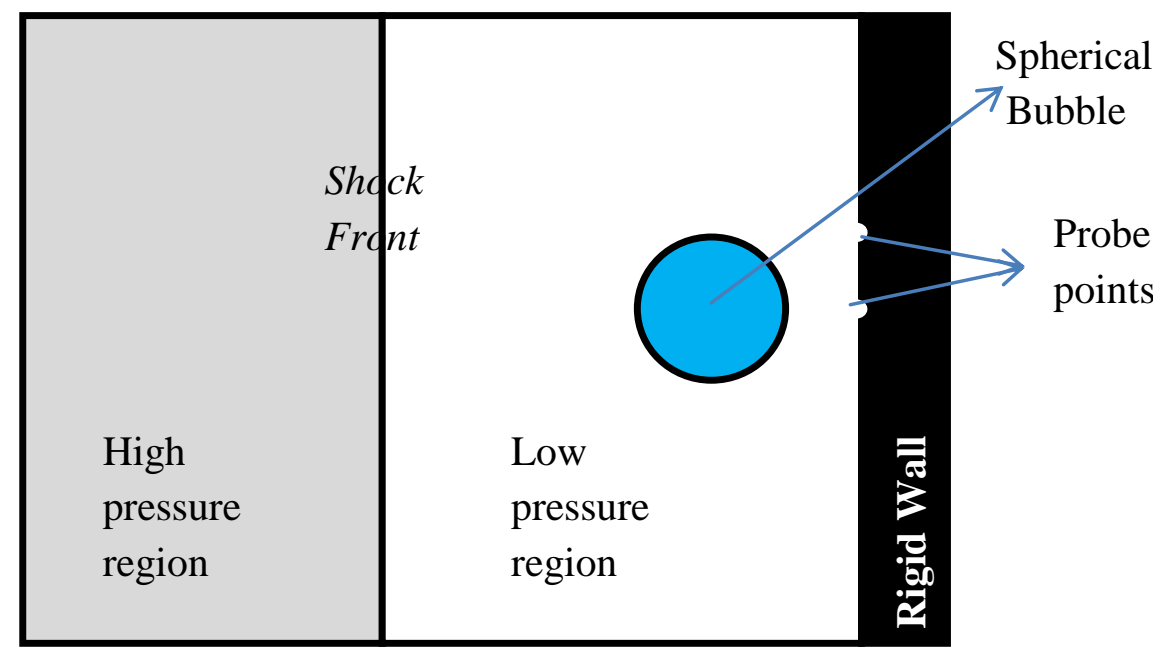

Figure 10. Schematic of the initial setup for shock/bubble interaction. A 35 MPa pressure wave in water on the left propagates towards an air bubble of radius $250 \mu \mathrm{m}$ residing at equilibrium in water at $0.1 \mathrm{MPa}$ ambient pressure. The bubble is located at a distance from the wall of $500 \mu m$. Two probe points at the wall (white dots) are monitored to record the pressure signal: one is aligned with the center of bubble and the other is a bubble radius $(250 \mu \mathrm{m})$ off-center.

As in the previous case, the planar shock wave hits the bubble resulting in a reflected expansion wave in water and a transmitted shock in air (Figure 24 (a)). The incoming shock wave also is diffracted as it interacts with the bubble and finally hits the rigid wall resulting in a reflected shock wave. Also, as in the previous case, the shock wave deforms the surface of the bubble farthest from the wall during the bubble collapse resulting in the formation of a reentrant jet towards the wall as shown in Figure 24 (b). Successive stages of the bubble collapse and rebound are shown in the bubble shapes in Figure 25. The collapse phase leading to bubble minimum is shown in Figure 25 (a-c) and the rebound phase leading to bubble fragmentation is shown in Figure 25 (d-f).

The velocity contours during reentrant jet evolution are shown in Figure 26 with the bubble finally deforming into a toroidal shape. 


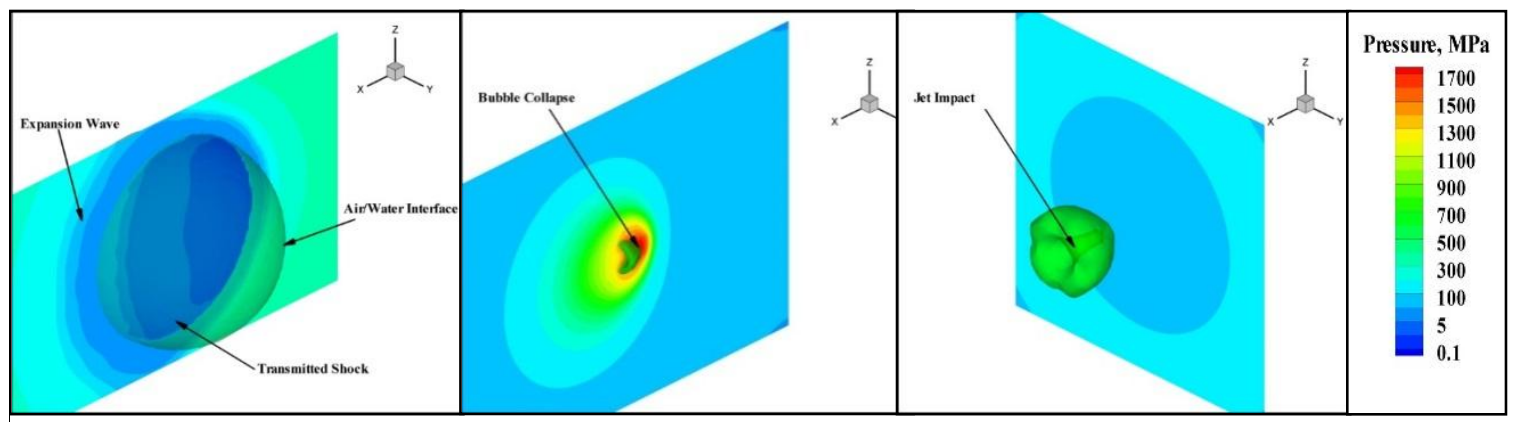

Figure 11. Dynamics of the interaction of a high pressure (35 MPa) shock wave in water and an air bubble of radius $250 \mu \mathrm{m}$ residing in equilibrium in water at a pressure of $0.1 \mathrm{MPa}$. Time sequence of various phases of the interaction: (a) Reflected expansion wave in the water and transmitted shock in the bubble, (b) Collapse with reentrant jet formation in the bubble, (c) impact of the reentrant jet on the other side of the bubble.
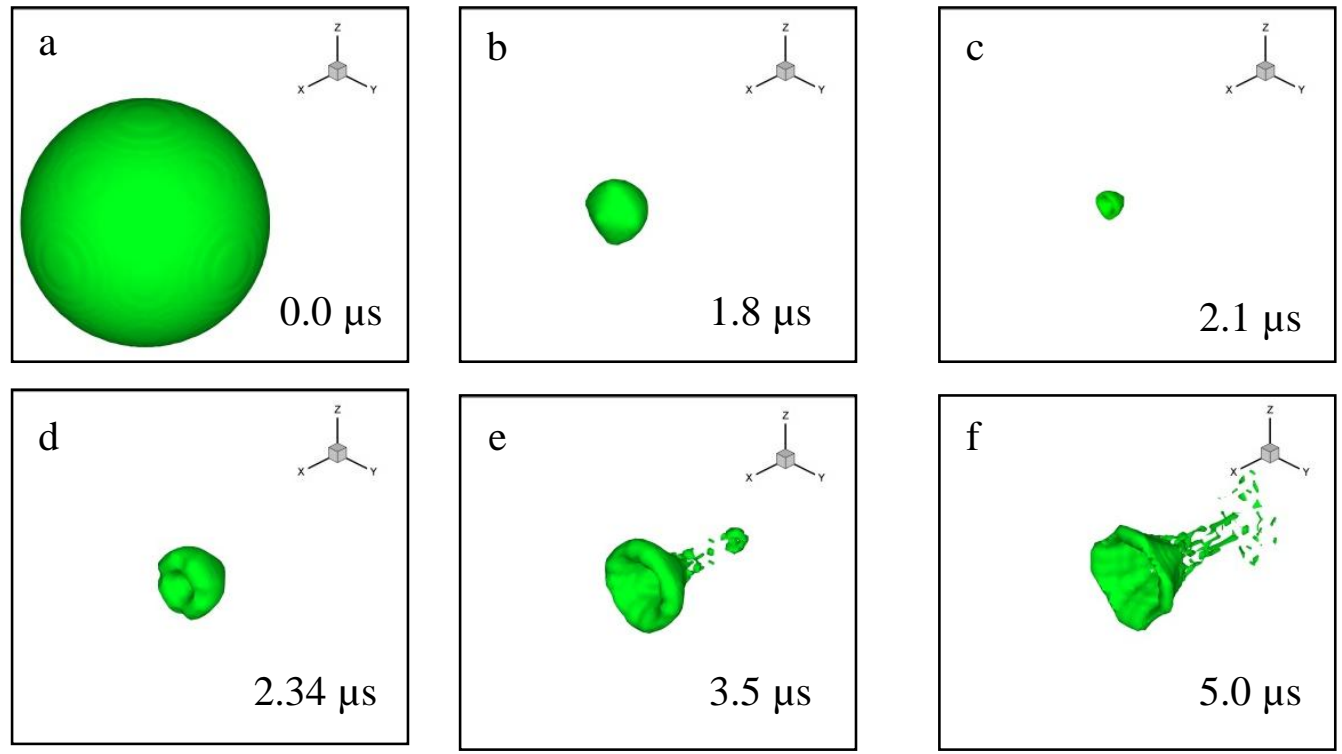

Figure 12. Dynamics of the interaction of a high pressure (35 MPa) shock wave in water and an air bubble of radius $250 \mu \mathrm{m}$ residing in equilibrium in water at a pressure of $0.1 \mathrm{MPa}$. Collapse and rebound phases of the interaction between a bubble and a shock wave. (a-c) Collapse phase leading to bubble minimum, (d-f) Rebound leading to bubble fragmentation. 


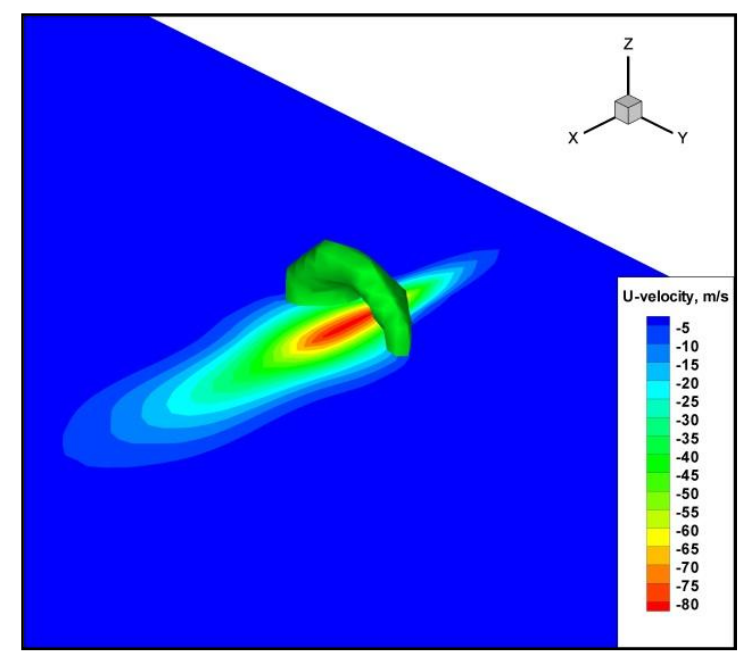

Figure 13. Dynamics of the interaction of a high pressure (35 MPa) shock wave in water and an air bubble of radius $250 \mu m$ residing in equilibrium in water at a pressure of 0.1 MPa. Snapshot of velocity contours depicting the reentrant jet dynamics.

The pressure at the wall is probed at two points, one at the axis of symmetry of the bubble and the other at a distance of one initial bubble radius $(250 \mu \mathrm{m})$ from the axis. The corresponding pressure histories at the wall clearly shows (Figure 27) a large increase in pressure as the bubble collapses, resulting from the bubble generated flow field. Both GEMINI and 3DYNAFS-COMP ${ }^{\odot}$ give almost identical results at the two observation points at the wall, indicating similar accuracy between 3DYNAFS-COMP ${ }^{\odot}$ and the Navy validated code [23]. 
a

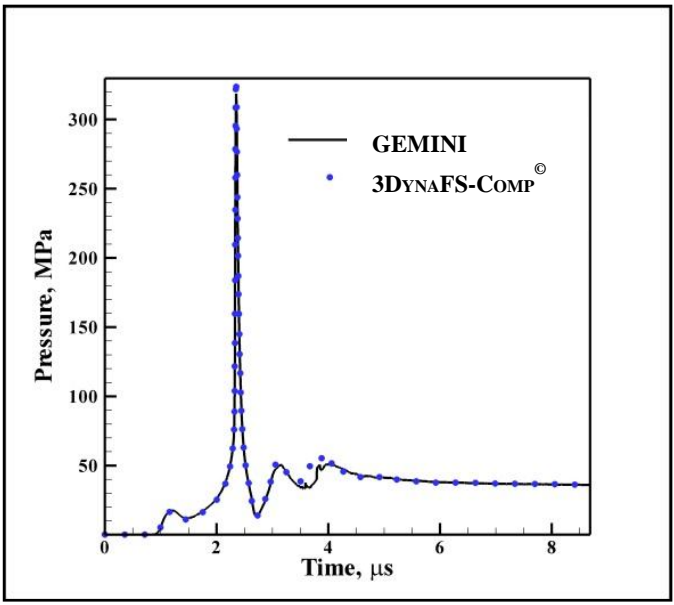

$\mathrm{b}$

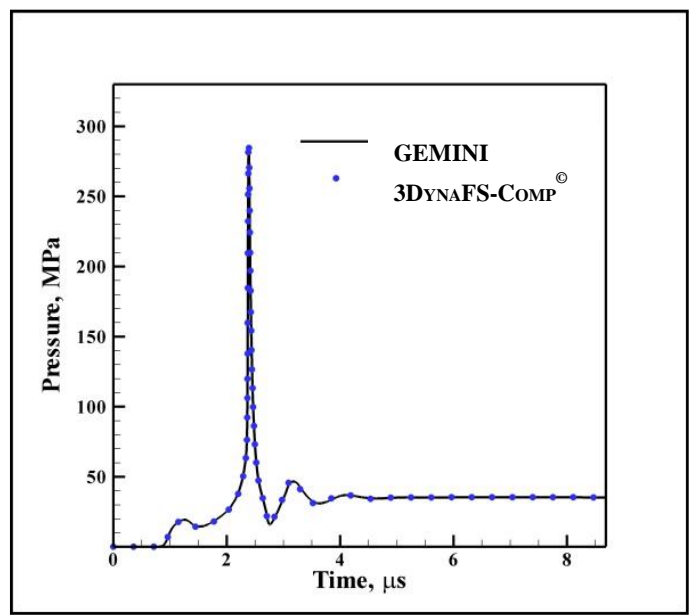

Figure 14. Comparison of the pressures versus time on the rigid wall between $3 D Y N A F S-C O M P^{\oplus}$ and the US Navy code GEMINI of at (a) the center of wall (b) at an off-centered point $250 \mu$ m away from the plate center. Dynamics of the interaction of a high pressure (35 MPa) shock wave in water and an air bubble of initial radius $250 \mu \mathrm{m}$ residing in equilibrium in water at a pressure of $0.1 \mathrm{MPa}$.

The evolution of the equivalent bubble radius (sphere with the same volume) is shown in Figure 28 (a). A clear decrease in the equivalent bubble radius is seen as the bubble is compressed by the incoming shock wave during its collapse. The bubble then rebounds as its inner pressure exceeds that of the surroundings and effectuates a couple of oscillations before settling to a balance radius of about $70 \mu \mathrm{m}$. The final pressure at the wall is close to $35 \mathrm{MPa}$ as the initial boundary conditions for the pressure in the far field are prescribed with $35 \mathrm{MPa}$.

These results compare again very well with GEMINI [23]. The comparison of the pressure evolution at the two probed points (Figure 27) and of the equivalent radius of bubble (Figure 28 (a)) shows an excellent agreement between the two codes. Figure 28(b) shows temporal variation in pressure at the wall and equivalent bubble radius. The offset in pressure peak and bubble minima indicates the time taken by the pressure wave to travel from the center of bubble to wall. The results are further compared by tracking two points on the bubble interface for both GEMINI and 3DYNAFS-COMP ${ }^{\circledR}$. Initially the points are located along the line perpendicular to the rigid wall and passing through the center of the bubble. The initial difference in location corresponds to bubble diameter $(500 \mu \mathrm{m})$ as shown in Figure 29. As the 
shock wave impacts the bubble interface, the interface deforms as shown by the tracked points in Figure 29. The implosion of the bubble interface occurs close to $2 \mu$ s resulting in conjunction of tracked points as seen in Figure 29. The results compared with GEMINI match perfectly with 3 DYNAFS-COMP ${ }^{\odot}$ until the conjunction of tracked points. 

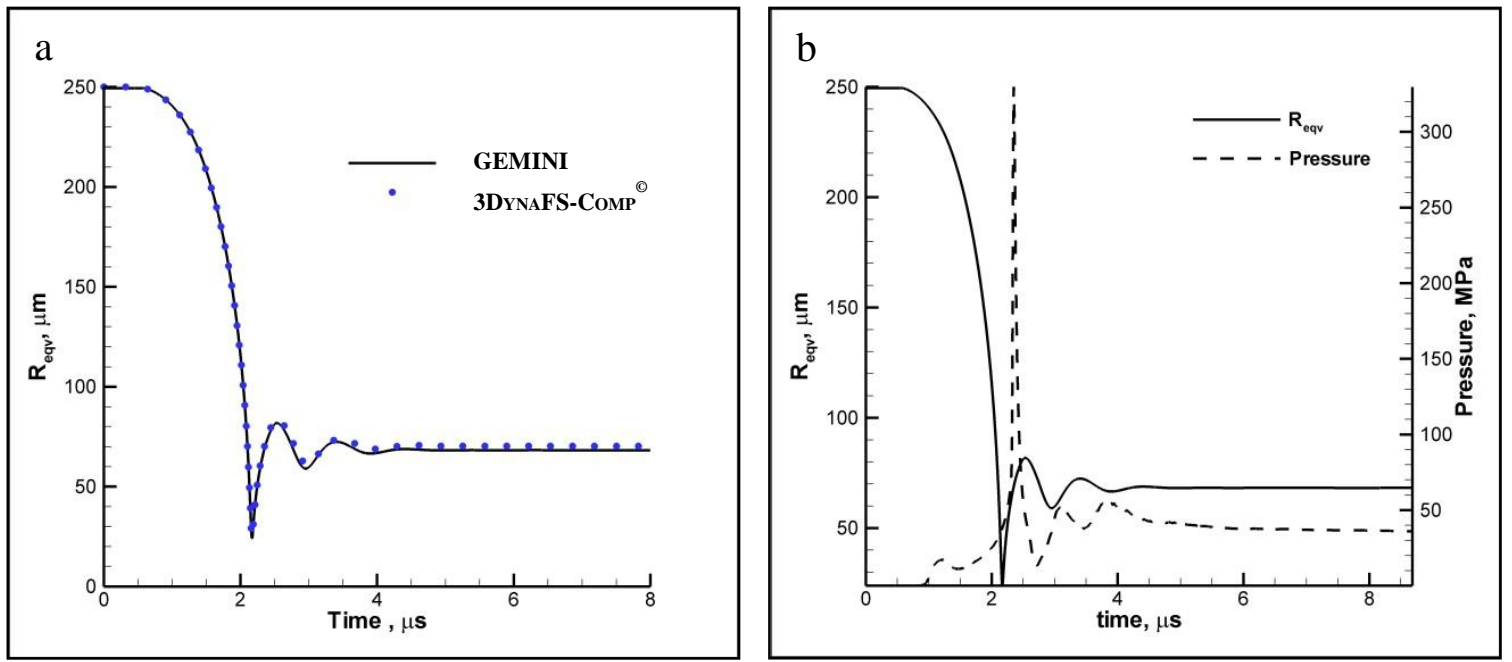

Figure 15. Dynamics of the interaction of a high pressure (35 MPa) shock wave in water and an air bubble of radius $250 \mu \mathrm{m}$ residing in equilibrium in water at a pressure of $0.1 \mathrm{MPa}$. a) Comparison of the time evolution of the bubble equivalent radius between $3 D Y N A F S-C O M P^{\odot}$ and GEMINI. b) Time evolution of equivalent bubble radius with pressure at the center of bubble.

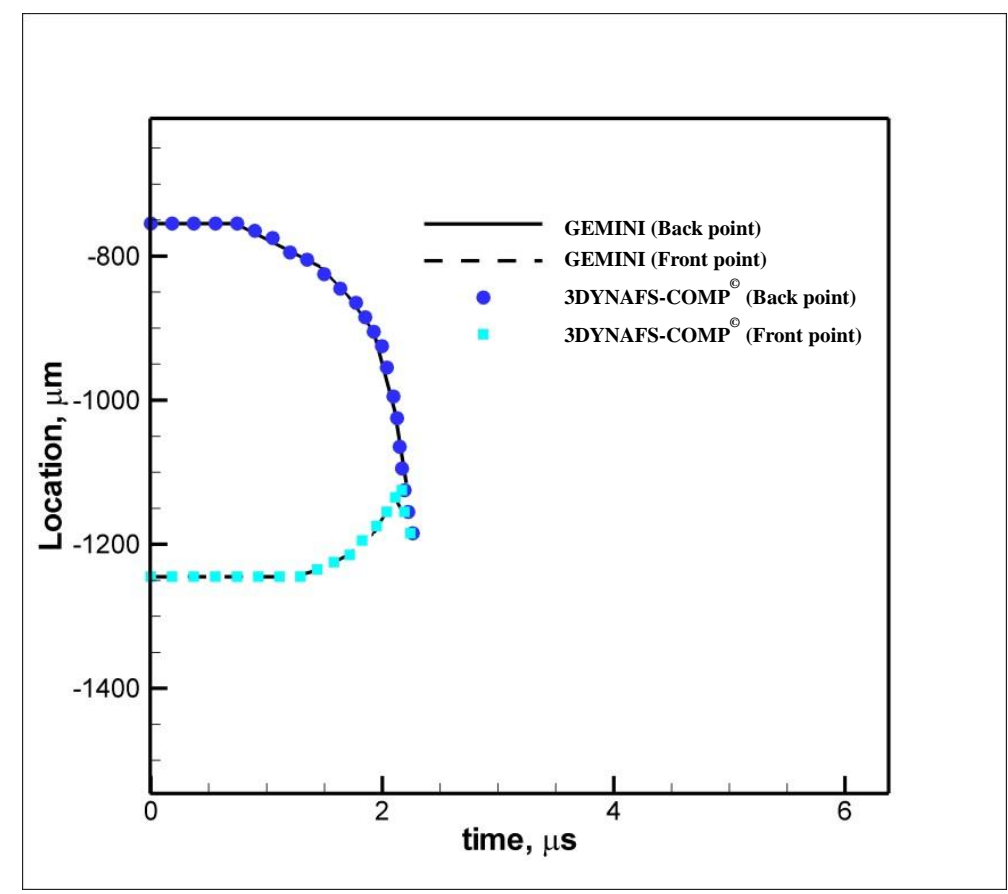

Figure 16. Comparison of interface dynamics between 3DYNAFS-COMP ${ }^{\oplus}$ and GEMINI by tracking two points (one in the front and other at the back) on the bubble interface Dynamics of the interaction of a high pressure (35 MPa) shock wave in water and an air bubble of radius $250 \mu \mathrm{m}$ residing in equilibrium in water at a pressure of $0.1 \mathrm{MPa}$. 


\subsection{Shock wave propagation in a bubbly medium}

The last example presented in this paper shows the propagation of a high-pressure wave in a two-phase medium including a large number of bubbles. When the shock wave encounters the array of bubbles in water it behaves very differently than a shock wave travelling in pure water. The presence of the bubbles attenuates significantly the wave amplitude. On the other hand, the collapses of the bubbles compressed by the shock wave can cause local pressure spikes in the bubble region. Such simulations are important in understanding initiation and safe handling of heterogeneous explosives and in modeling cavitation erosion. In the test case presented here, a shock wave of amplitude $35 \mathrm{MPa}$ interacts with a layer of bubbles residing in water at an ambient pressure of 0.1 MPa. The geometry is illustrated in Figure 30. The dimensions of the computational domain are $2 \mathrm{~cm} \times 0.5 \mathrm{~cm} \times 0.5 \mathrm{~cm}$ and the initial radius of the bubbles is $500 \mu \mathrm{m}$. A uniform grid of $50 \mu \mathrm{m}$ is used for this problem. A 3D block of the fluid is modelled and 24 bubbles are included in the bubbly region of length $0.3 \mathrm{~cm}$.

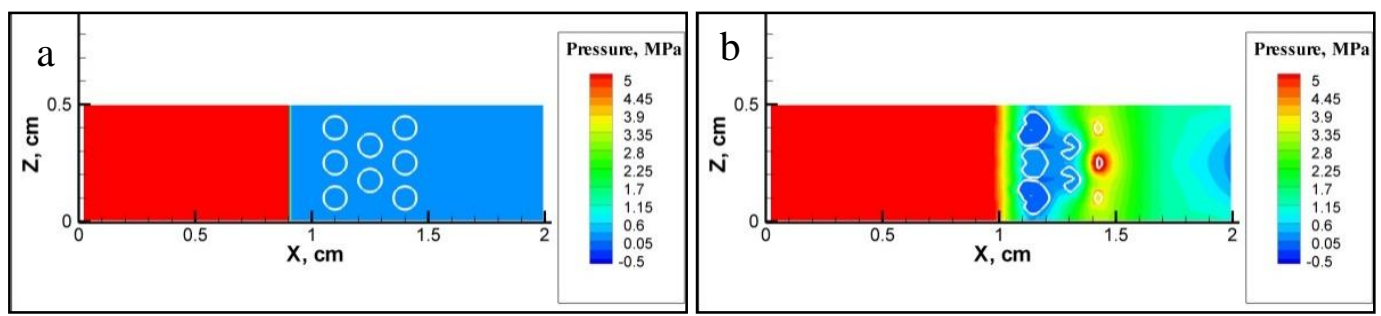

Figure 30. (a) Initial setup of shock wave interaction with multiple bubbles. A shock wave of strength 35 MPa propagates and interacts with a bubbly layer in the region $X=[0.011 \mathrm{~m}, 0.014 \mathrm{~m}]$. The bubble radii are $500 \mu m$, and 24 bubbles are involved in the computation. (b) Pressures and bubbles distributions at a selected time during the computations, $t=31 \mu \mathrm{s}$.

The pressure is monitored along a line on the $\mathrm{X}$-axis passing through the center of a $\mathrm{Y}=$ constant plane (here, $\mathrm{Y}=0.25 \mathrm{~cm}$ ) of the computational domain. The pressure is monitored at several locations such as in Figure 31(a) in a region upstream of the bubbly layer, (b) 
inside the bubbly layer and (c) downstream of the bubbly layer. Figure 31(a) shows how, upstream of the bubbly layer, the observation points see the pressure decay following the 35 MPa wave passage, followed by an expansion wave due to reflection from the bubbly layer interface.
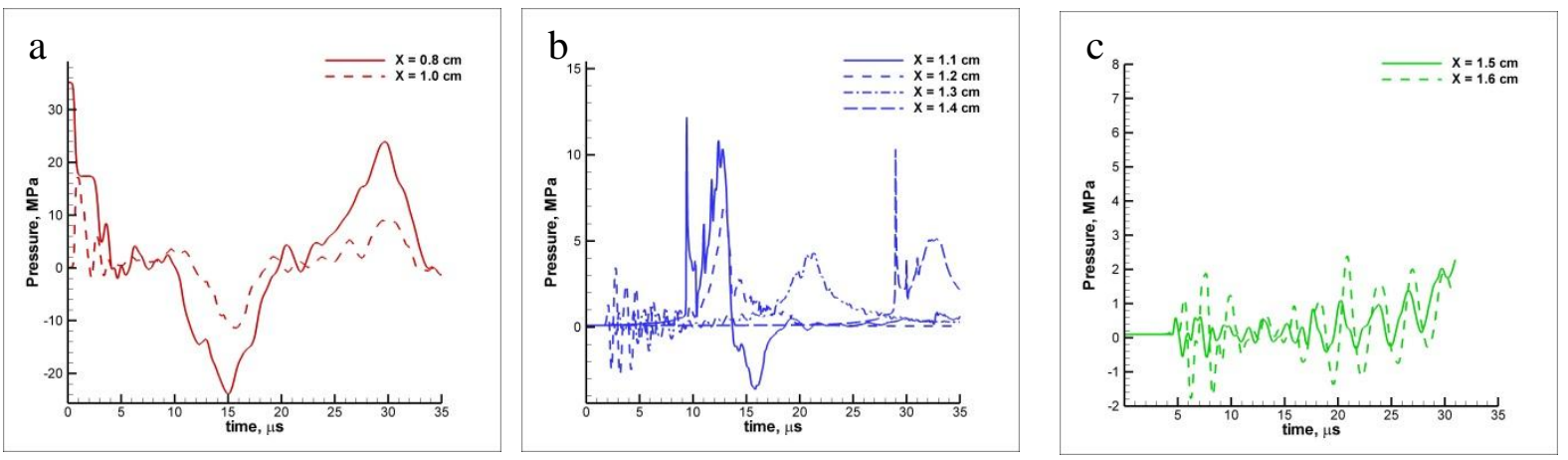

Figure 31. Pressure monitored along a line along $X$-axis at the center of plane $Y=0.25 \mathrm{~cm}$. (a) monitored points upstream the bubbly layer (b) monitored points inside the bubbly layer (c) monitored points downstream the bubbly layer.

Inside the bubbly layer (Figure 30(b) and Figure 31(b)) the pressure shows the formation of local hot pressure spots with sudden rises in the pressure, for example at $t=31 \mu$ s at $x=1.1 \mathrm{~cm}$. The strong attenuation of wave is clearly observed in Figure 31(c) at locations outside the layer where the pressure does not exceed $2 \mathrm{MPa}$ despite the 35Mpa imposed shock. The exercise indicates that the current algorithm is robust and can handle multiple interfaces in the presence of high pressure shock wave.

\section{Conclusion}

An Eulerian-Lagrangian approach to handle the high-speed dynamics of multi-material interfaces is presented. The method combines good features from both Eulerian and Lagrangian frameworks by treating the computational cells describing the interfaces in a fully conservative Eulerian-Lagrangian manner and cells away from the interface in a fixed grid 
Eulerian frame. This combined procedure obviates the issue involving mesh entanglement faced in Lagrangian flow solvers and does not require population of an Eulerian ghost field to compute fluxes across the interfaces, which is non-conservative.

The method was demonstrated here on idealized shock tube and Riemann problems and has shown an excellent agreement with standard analytical cases involving both strong and weak shock problems. The approach was also demonstrated as robust in handling shock/bubble interaction problems such as in underwater explosions, two-phase flows, or cavitation scenarios and matches well with similar previously published results in the literature. This framework shows strong capability of application to a variety of interfacial interactions problems involving solid-fluid, solid-solid, and liquid-gas problems.

\section{Acknowledgments}

This work was conducted under partial funding from DOE SBIR Phase II DE-FG0207ER84839, DOE SBIR Phase II DE-SC0006443 and from DYNAFLOW, INC. internal funding. We acknowledge gratefully the support of both organizations.

\section{References}

1. Lax, P.D. and X.-D. Liu, Solution of two-dimensional Riemann problems of gas dynamics by positive schemes. SIAM Journal on Scientific Computing, 1998. 19(2): p. 319-340.

2. Cullis, I., Blast waves and how they interact with structures. Journal of the Royal Army Medical Corps, 2001. 147(1): p. 16-26.

3. Chahine, G., et al. Spark-generated bubbles as laboratory-scale models of underwater explosions and their use for validation of simulation tools. in Proceedings of the 66th Shock and Vibration Symposium. 1995. Biloxi, MS. 
4. Kapahi, A., S. Sambasivan, and H. Udaykumar, A three-dimensional sharp interface Cartesian grid method for solving high speed multi-material impact, penetration and fragmentation problems. Journal of Computational Physics, 2013.

5. Baer, M. and J. Nunziato, A two-phase mixture theory for the deflagration-to-detonation transition (DDT) in reactive granular materials. International journal of multiphase flow, 1986. 12(6): p. 861-889.

6. Camacho.G.T. and M.Ortiz., Adaptive lagrangian modelling of ballistic penetration of metallic targets. Comput. Methods Appl. Mech. Eng., 1997. 142(3-4): p. 269-301.

7. Xiao, H., O. Bruhns, and A. Meyers, Elastoplasticity beyond small deformations. Acta Mechanica, 2006. 182(1): p. 31-111.

8. Sambasivan, S., A. Kapahi, and H.S. Udaykumar, Simulation of high speed impact, penetration and fragmentation problems on locally refined Cartesian grids. Journal of Computational Physics, 2013. 235: p. 334-370.

9. Johnson, G.R. and W.H. Cook, Lagrangian EPIC code computations for oblique, yawed-rod impacts onto thin-plate and spaced-plate targets at various velocities. International Journal of Impact Engineering, 1993. 14(1): p. 373-383.

10. Hallquist, J.O., LS-DYNA users manual. Livermore software technology corporation, 2000.

11. Durrett, R.E. and D.A. Matuska, The HULL Code, Finite Difference Solution to the Equations of Continuum Mechanics. Air Force Armament Laboratory, AFATL-TR-78-125, 1978.

12. McGlaun, J.M., S. Thompson, and M. Elrick, CTH: a three-dimensional shock wave physics code. International Journal of Impact Engineering, 1990. 10(1): p. 351-360.

13. Anderson, C.E., An overview of the theory of hydrocodes. International Journal of Impact Engineering., 1987. 5(1-4): p. 33-59.

14. Kapahi, A., J. Mousel, S. Sambasivan, and H. Udaykumar, Parallel, sharp interface Eulerian approach to high-speed multi-material flows. Computers \& Fluids, 2012.

15. Kapahi, A., S. Sambasivan, and H. Udaykumar, Simulation of collapse and fragmentation phenomena in a sharp interface Eulerian setting. Computers \& Fluids, 2012. 
16. Benson, D., Computational methods in Lagrangian and Eulerian hydrocodes. Computer methods in Applied mechanics and Engineering, 1992. 99(2-3): p. 235-394.

17. Zhou, C., G. Liu, and K. Lou, Three-dimensional penetration simulation using smoothed particle hydrodynamics. International Journal of Computational Methods, 2007. 4(4): p. 671692.

18. Maire, P.H., et al. A cell-centered Arbitrary Lagrangian Eulerian (ALE) method for multimaterial compressible flows. in ESAIM: Proceedings. 2008.

19. Luo, H., J.D. Baum, and R. Löhner, On the computation of multi-material flows using ALE formulation. Journal of Computational Physics, 2004. 194(1): p. 304-328.

20. Souli, M., A. Ouahsine, and L. Lewin, ALE formulation for fluid-structure interaction problems. Computer methods in applied mechanics and engineering, 2000. 190(5-7): p. 659675.

21. Lomtev, I., R.M. Kirby, and G.E. Karniadakis, A discontinuous Galerkin ALE method for compressible viscous flows in moving domains. Journal of Computational Physics, 1999. 155(1): p. 128-159.

22. Colella, P., A direct Eulerian MUSCL scheme for gas dynamics. SIAM Journal on Scientific and Statistical Computing, 1985. 6(1): p. 104-117.

23. Wardlaw, A., et al., The Gemini Coupled Hydrocode. Indian Head Div., Naval Surface Warfare Center, Report IHTR, 2003. 2500.

24. Saurel, R. and R. Abgrall, A multiphase Godunov method for compressible multifluid and multiphase flows. Journal of Computational Physics, 1999. 150(2): p. 425-467.

25. Raju, R., et al., Study of Pressure Wave Propagation in a Two-Phase Bubbly Mixture. Journal of Fluids Engineering, 2011. 133(12): p. 121302-1-121302-12.

26. Toro, E.F., Riemann solvers and numerical methods for fluid dynamics: a practical introduction. 2009: Springer.

27. Arun, K. R., Medvidova, M. L., Prasad, P., \& Rao, S. V. R. (2013). Recent Developments in the Numerics of Nonlinear Hyperbolic Conservation Laws. Notes on Numerical Fluid Mechanics and Multidisciplinary Design, 120, 1-24. doi:10.1007/978-3-642-33221-0 
28. Anderson, J.D., Modern compressible flow: with historical perspective. Vol. 12. 1990: McGraw-Hill New York.

29. Zel'Dovich, Y.B. and Y.P. Raizer, Physics of shock waves and high-temperature hydrodynamic phenomena. 2002: Dover Pubns.

30. Osher, S. and S. Chakravarthy, Upwind schemes and boundary conditions with applications to Euler equations in general geometries. Journal of Computational Physics, 1983. 50(3): p. $447-481$.

31. Laney, C.B., Computational gasdynamics. 1998: Cambridge University Press.

32. Van Leer, B., Towards the ultimate conservative difference scheme. V. A second-order sequel to Godunov's method. Journal of Computational Physics, 1979. 32(1): p. 101-136.

33. Colella, P., H.M. Glaz, and R.E. Ferguson, Multifluid algorithms for Eulerian finite difference methods, U.o. Berkley, Editor. 1990: Unpublished manuscript.

34. Noh, W.F. and P. Woodward. SLIC (simple line interface calculation). in Proceedings of the Fifth International Conference on Numerical Methods in Fluid Dynamics June 28-July 2, 1976 Twente University, Enschede. 1976. Springer.

35. Arienti, M. and J.E. Shepherd, A numerical study of detonation diffraction. Journal of Fluid Mechanics, 2005. 529(2005): p. 117-146.

36. Hawker, N.A. and Y. Ventikos, Interaction of a strong shockwave with a gas bubble in a liquid medium: a numerical study. Journal of Fluid Mechanics, 2012. 701: p. 59-97.

37. Kapahi, A. and H. Udaykumar, Dynamics of void collapse in shocked energetic materials: physics of void-void interactions. Shock Waves, 2013: p. 1-22.

38. Chahine, G.L. and T.O. Perdue. Simulation of the three-dimensional behavior of an unsteady large bubble near a structure. in AIP Conference Proceedings. 1990.

39. Field, J., The physics of liquid impact, shock wave interactions with cavities, and the implications to shock wave lithotripsy. Physics in medicine and biology, 1991. 36(11): p. 1475. 